九州大学学術情報リポジトリ

Kyushu University Institutional Repository

\title{
Affine vertex operator algebras and modular linear differential equations
}

Arike, Yusuke

Division of Mathematics, Faculty of Pure and Applied Sciences, University of Tsukuba

Kaneko, Masanobu

Faculty of Mathematics, Kyushu University : Professor

Nagatomo, Ki yokazu

Department of Pure and Applied Mathematics, Graduate School of Information Science and Technology, Osaka University

Sakai, Yuichi

http://hdl. hand le. net/2324/4753038

出版情報: Letters in Mathematical Physics. 106，pp.693-718，2016-04-12. Springer バージョン：

権利関係 : 


\title{
Affine vertex operator algebras and modular linear differential equations
}

\author{
Yusuke Arike $^{1)}$, Masanobu Kaneko ${ }^{2)}$, Kiyokazu Nagatomo ${ }^{3)}$ and Yuichi Sakai ${ }^{4)}$ \\ 1) Division of Mathematics, \\ Faculty of Pure and Applied Sciences, \\ University of Tsukuba, Tsukuba, Ibaraki 305-8571, JAPAN \\ e-mail: arike@math.tsukuba.ac.jp \\ 2) Faculty of Mathematics, Kyushu University \\ Fukuoka 819-0395, JAPAN \\ email: mkaneko@math.kyushu-u.ac.jp \\ 3) Department of Pure and Applied Mathematics \\ Graduate School of Information Science and Technology \\ Osaka University, Toyonaka, Osaka 560-0043, JAPAN \\ e-mail: nagatomo@math.sci.osaka-u.ac.jp \\ 4) Yokomizo 3012-2, Oki-machi, Mizumagun, Fukuoka 830-0405, JAPAN \\ e-mail: dynamixaxs@gmail.com
}

\begin{abstract}
In this paper we list all affine vertex operator algebras of positive integral levels whose dimensions of spaces of characters are at most 5 and show that a basis of the space of characters of each affine vertex operator algebra in the list gives a fundamental system of solutions of a modular linear differential equation. Further we determine the dimensions of the spaces of characters of affine vertex operator algebras whose numbers of inequivalent simple modules are not exceeding 20.
\end{abstract}

AMS Subject Classification 2010: Primary 11F11, 81T40, Secondary 17B69

Key words: Affine vertex operator algebra, Modular invariance, Modular linear differential equation, 2-dimensional conformal field theory

\section{Introduction}

A modular linear differential equation (MLDE for short) of weight $k$ is a linear differential equation $\vartheta_{k}^{n} f+\sum_{j=0}^{n-1} P_{2(n-j)} \vartheta_{k}^{j} f=0$, where $\vartheta_{k}$ is the Serre derivation of weight $k$ and $P_{2 j}$ is a classical modular form of weight $2 j$. This has a regular singularity at $q=0$ where $q=e^{2 \pi i \tau}$. The MLDEs appear and play important roles in 2-dimensional conformal field theory and number theory. In [15], S. D. Mathur, S. Mukhi and A. Sen classified rational conformal field theories whose partition functions (characters) satisfy 2nd order MLDEs. On the one hand, in number theory, M. Kaneko and D. Zagier ([12]) introduced 2nd order MLDEs (called 
Kaneko-Zagier equation) in the study of supersingular $j$-invariants of elliptic curves. These two 2nd order MLDEs were proved to be equivalent in [11].

In this paper we study some connections between MLDEs and vertex operator algebras (which are the mathematical counterpart of conformal field theories). One of the most important features in the theory of vertex operator algebras (VOAs) is the modular invariance property of characters of simple modules ([19]). The character of a simple module $M$ of a VOA is defined by $\operatorname{tr}_{M} q^{L_{0}-c / 24}\left(q=e^{2 \pi i \tau}\right)$, where $c$ is the central charge of $V$ and $L_{0}$ is the grading operator. In [19] it was shown that if a VOA is $C_{2}$-cofinite and rational, then there is a linear differential equation with modular coefficients that every character satisfies. This linear differential equation was shown to be a MLDE in [2, Lemma 6.3]. By using this result it was shown that any character of a simple module converges on the upper-half plane and the space of characters is invariant under the slash action with weight 0 of $S L_{2}(\mathbb{Z})$. In other words, the characters of simple modules form a vector-valued modular form of weight 0 on $S L_{2}(\mathbb{Z})$.

It is known in cases of several affine VOAs ([15]) and Virasoro minimal models ([3, 4, $16,17])$ that there are MLDEs whose spaces of solutions coincide with those of characters. However, since any solution of the 1st order MLDE of weight 0 is constant, the character of a VOA with a unique simple module (such VOAs are called holomorphic in the theory of VOAs) does not satisfy the 1 st order MLDE of weight 0 . For instance, the affine VOA $L_{E_{8}, 1}$ associated with the finite-dimensional simple Lie algebra of type $E_{8}$ of level 1 has a unique simple module whose character is $j(\tau)^{1 / 3}$, where $j(\tau)$ is the $j$-function. It is known in [15] (also see [11]) that the character of $L_{E_{8}, 1}$ satisfies the 2nd order MLDE of weight 0. Another example is the affine VOA $L_{A_{2}, 3}$ associated with the finite-dimensional simple Lie algebra of type $A_{2}$ of level 3 . The number of inequivalent simple $L_{A_{2}, 3}$-modules is 10 and the dimension of the space of characters is 6 . However, any basis of the space of characters of $L_{A_{2}, 3}$ does not form a fundamental system of solutions of any 6th order MLDE of weight 0 (see Table 4 in $\S 4$ ). Therefore it seems to be natural to ask if the space of characters of a VOA has a MLDE whose fundamental system of solutions is given by a basis of the space of characters.

The VOAs appeared in the classification given in [15] are all affine VOAs of level 1 associated with finite-dimensional simple Lie algebras of the Deligne exceptional series ([1]). Hence, motivated by this fact, we intensively study spaces of characters of rational and $C_{2}$-cofinite affine VOAs. Let $\mathfrak{g}$ be a complex finite-dimensional simple Lie algebra, $\hat{\mathfrak{g}}$ the associated affine Lie algebra, and $L_{\mathfrak{g}, k}$ the irreducible integrable highest weight $\hat{\mathfrak{g}}$-module with highest weight 0 of positive integral level $k$. Then $L_{\mathfrak{g}, k}$ is a rational and $C_{2}$-cofinite VOA and $L_{\mathfrak{g}, k}(\Lambda)$, where $\Lambda$ is a dominant integral weight of level $k$, forms the complete set of inequivalent simple $L_{\mathfrak{g}, k}$-modules ([5]). In this paper we determine the dimension of the space of characters of $L_{\mathfrak{g}, k}$ with at most 20 simple modules, and we find that the affine VOAs whose bases of the spaces of characters give fundamental systems of solutions of MLDEs if the dimensions of the spaces of characters are at most 5 (except for $L_{E_{8}, 1}$ ).

An integrable highest weight irreducible $\hat{\mathfrak{g}}$-module $L_{\mathfrak{g}, k}(\Lambda)$ is a module of the Virasoro algebra of central charge $c_{\mathfrak{g}, k}=k \operatorname{dim} \mathfrak{g} / 2\left(k+h^{\vee}\right)$ by the Sugawara construction (see $\left.[8,5]\right)$, where $h^{\vee}$ is the dual Coxeter number. The $\hat{\mathfrak{g}}$-module $L_{\mathfrak{g}, k}(\Lambda)$ is decomposed into a direct sum of finite-dimensional eigenspaces of $L_{0}$. Then there is a rational number $h_{\Lambda}$ such that $L_{0}$-eigenvalues of $L_{\mathfrak{g}, k}$ is included in the set $h_{\Lambda}+\mathbb{Z}_{\geq 0}$ and $h_{\Lambda}$ is called the conformal weight 
of $L_{\mathfrak{g}, k}(\Lambda)$. The lowest power in $q$ of the character of $L_{\mathfrak{g}, k}(\Lambda)$ is $h_{\Lambda}-c_{\mathfrak{g}, k} / 24$. Therefore, if the conformal weights are mutually distinct, by the definition of characters, the dimension of the space of characters coincides with the number of inequivalent simple modules. It happens that inequivalent simple modules have the same conformal weight. Then we may use diagram automorphisms of affine Lie algebras and the theory of $S$-matrix to check if the corresponding characters are linear independent or not.

In [14] G. Mason introduced the concept of modular Wronskian of vector-valued modular forms on $S L_{2}(\mathbb{Z})$. It is also proved that component functions of a vector-valued modular form constitute a fundamental system of a MLDE if and only if the modular Wronskian never vanishes on the upper half-plane, which we call non-zero Wronskian condition. In many situations this condition is confirmed by looking at several powers in $q$ of each character. Particularly, it is not hard to check non-zero Wronskian condition if an affine VOA has mutually distinct conformal weights. It is worthy to say that many affine VOAs have mutually distinct conformal weights.

The paper is organized as follows. In $\S 1$ we recall definitions of vector-valued modular forms, modular linear differential equations and non-zero Wronskian condition. In $\S 2$ we review the theory of affine Lie algebras and affine VOAs. Several relations between characters and diagram automorphisms are also discussed here. The classification of affine VOAs by the dimensions of the spaces of characters (up to dimension 5 ) is given in $\S 3$. We also take account of non-zero Wronskian condition for these affine VOAs. In $\S 4$ we give lists of the dimensions of the spaces of characters of affine VOAs with at most 20 inequivalent simple modules and non-zero Wronskian condition. The dimensions of characters and non-zero Wronskian condition are mostly affirmed only by the information of conformal weights and diagram automorphisms. In some cases we additionally use the action of the transformation $\tau \mapsto-1 / \tau$ $(S$-matrices) as being done in $\S 5$.

In this research we often used the program "kac" given by B. Schellekens who kindly gave several introductions to the 3rd author K. N.

\section{Vector-valued modular forms and modular linear differential equations}

In this paper $\Gamma_{1}$ always represents the (full) modular group $S L_{2}(\mathbb{Z})$ with generators

$$
S=\left(\begin{array}{cc}
0 & -1 \\
1 & 0
\end{array}\right) \quad \text { and } \quad T=\left(\begin{array}{ll}
1 & 1 \\
0 & 1
\end{array}\right)
$$

and holomorphic modular forms on $\Gamma_{1}$ are called classical modular forms.

According to [14] we recall the concept of vector-valued modular forms. Let $\mathbb{H}$ be the complex upper half-plane and $\mathcal{F}$ the space of holomorphic functions on $\mathbb{H}$, and $\mathbb{F}={ }^{t}\left(f_{1}, \ldots, f_{n}\right)$ a column vector whose entries are elements in $\mathcal{F}$. For a given integer $k$ the weight $k$ slash action $\left.\right|_{k} \gamma$ of $\Gamma_{1}$ on $\mathbb{F}$ is defined by $\left.\mathbb{F}\right|_{k} \gamma={ }^{t}\left(\left.f_{1}\right|_{k} \gamma, \ldots,\left.f_{n}\right|_{k} \gamma\right)$ for every $\gamma \in \Gamma_{1}$, where

$$
\left.f\right|_{k} \gamma=(c \tau+d)^{-k} f(\gamma(\tau)), \quad \gamma=\left(\begin{array}{ll}
a & b \\
c & d
\end{array}\right) \in \Gamma_{1} .
$$


Let $\rho: \Gamma_{1} \rightarrow G L_{n}(\mathbb{C})$ be an $n$-dimensional complex representation of $\Gamma_{1}$. Then we have a right action of $\Gamma_{1}$ on $\mathcal{F}^{n}$ by $\left.\mathbb{F} \mapsto \rho(\gamma)^{-1} \mathbb{F}\right|_{k} \gamma$ for every $\gamma \in \Gamma_{1}$. A weak vector-valued modular form of weight $k$ is a $\Gamma_{1}$-invariant vector-valued function with respect to this action.

Definition. Let $\mathbb{F}={ }^{t}\left(f_{1}, \ldots, f_{n}\right)$ be a weak vector-valued modular form of weight $k$. Then $\mathbb{F}$ is called a (meromorphic) vector-valued modular form of weight $k$ if each component function $f_{j}$ has a $q$-expansion which is convergent in a neighborhood of the infinity $i \infty$;

$$
f_{j}(\tau)=q^{\lambda_{j}} \sum_{n=0}^{\infty} a_{n, j} q^{n}, \lambda_{j} \in \mathbb{R}, q=e^{2 \pi i \tau}(\tau \in \mathbb{H}) .
$$

If $\lambda_{j}$ is non-negative we say that $f_{j}$ is holomorphic at the infinity, and if each $f_{j}$ satisfies this condition then $\mathbb{F}$ is called a holomorphic vector-valued modular form.

Remark. Let ${ }^{t}\left(f_{1}, \ldots, f_{n}\right)$ be a vector-valued modular form of weight $k$. Then the space spanned by $f_{1}, \ldots, f_{n}$ is a $\Gamma_{1}$-module by the slash action $\left.\right|_{k}$.

Let $\rho$ be a complex representation of $\Gamma_{1}$. Let $\mathbb{M}_{k}(\rho)$ and $\mathbb{H}_{k}(\rho)$ denote the associated spaces of meromorphic and holomorphic vector-valued modular forms of weight $k$, respectively, and set $\mathbb{M}(\rho)=\bigoplus_{k \in \mathbb{Z}} \mathbb{M}_{k}(\rho)$ and $\mathbb{H}(\rho)=\bigoplus_{k \in \mathbb{Z}} \mathbb{H}(\rho)$. If $\rho$ is a trivial representation $\mathbf{1}$, then $\mathbb{H}(\mathbf{1})$ is a ring of classical modular forms on $\Gamma_{1}$.

Let ${ }^{t}\left(f_{1}, \ldots, f_{n}\right) \in \mathbb{M}_{k}(\rho)$. If $f_{1}, \ldots, f_{n}$ are not linearly independent, then there is a basis $\left\{g_{1}, \ldots, g_{m}\right\}$ of the $\Gamma_{1}$-module $\left\langle f_{1}, \ldots, f_{n}\right\rangle_{\mathbb{C}}$ and a unique (up to equivalence) representation $\rho^{\prime}: \Gamma_{1} \rightarrow G L_{m}(\mathbb{C})$ such that ${ }^{t}\left(g_{1}, \ldots, g_{m}\right) \in \mathbb{M}_{k}\left(\rho^{\prime}\right)$.

Every $\mathbb{F}={ }^{t}\left(f_{1}, \ldots, f_{n}\right) \in \mathbb{M}_{k}(\rho)$ is called normalized if there is an integer $0 \leq r \leq n$ such that

$$
f_{j}(\tau)=q^{\lambda_{j}}+\cdots(1 \leq j \leq r), \quad \lambda_{1}>\lambda_{2}>\ldots>\lambda_{r}, \quad f_{j}=0(r+1 \leq j \leq n) .
$$

For any $\mathbb{F} \in \mathbb{M}_{k}(\rho)$ there is an invertible matrix $A \in G L_{n}(\mathbb{C})$ such that $A \mathbb{F}$ is normalized. We call $A \mathbb{F}$ a normalized form of $\mathbb{F}$. It is obvious that component functions $f_{1}, \ldots, f_{n}$ of a vector-valued modular form ${ }^{t}\left(f_{1}, \ldots, f_{n}\right) \in \mathbb{M}_{k}(\rho)$ are linearly independent if and only if any entry of a normalized form is not 0 (equivalently $n=r$ in (1)).

Let $\vartheta_{k}$ be the Serre derivation acting on meromorphic functions on $\mathbb{H}$;

$$
\vartheta_{k}=q \frac{d}{d q}-\frac{k}{12} E_{2},
$$

where $E_{2}$ is the (quasimodular) Eisenstein series of weight 2;

$$
E_{2}(\tau)=1-24 \sum_{n=1}^{\infty}\left(\sum_{d \mid n} d\right) q^{n} .
$$

It obviously follows that $\vartheta_{k}\left(\left.f\right|_{k} \gamma\right)=\left.\left(\vartheta_{k} f\right)\right|_{k+2} \gamma$ for every $f \in \mathcal{F}$ and $\gamma \in \Gamma_{1}$ and then that each $\vartheta_{k}$ defines a linear map $\vartheta_{k}: \mathbb{M}_{k}(\rho) \rightarrow \mathbb{M}_{k+2}(\rho)$, where $\rho$ is a representation of $\Gamma_{1}$ on $G L_{n}(\mathbb{C})$. We define $\vartheta_{k}^{n}=\vartheta_{k+2(n-1)} \circ \cdots \vartheta_{k+2} \circ \vartheta_{k}$ for a positive integer $n$. Let $\mathbb{F}={ }^{t}\left(f_{1}, \ldots, f_{n}\right)$ be a meromorphic vector-valued modular form of weight $k$. The modular Wronskian $W(\mathbb{F})$ introduced in [14] is the $n \times n$ determinant given by column vectors as $W(\mathbb{F})=\operatorname{det}\left(\mathbb{F}, \vartheta_{k} \mathbb{F}, \ldots, \vartheta_{k}^{n-1} \mathbb{F}\right)$. It is proved in $[14$, Lemma 3.6] that $W(\mathbb{F})$ does not vanish identically if and only if $f_{1}, \ldots, f_{n}$ are linearly independent as usual Wronskian. 
Theorem 1 ([14, Theorem 3.7]). Let $\mathbb{F}={ }^{t}\left(f_{1}, \ldots, f_{n}\right) \in \mathbb{M}_{k}(\rho)$. Suppose that $f_{1}, \ldots, f_{n}$ are linearly independent and normalized as (1). Set $\lambda=\lambda_{1}+\cdots+\lambda_{n}$. Then there is a classical modular form $G \in \mathbb{H}_{n(n+k-1)-12 \lambda}(\mathbf{1})$ such that $G(i \infty) \neq 0$ and $W(\mathbb{F})=G \eta^{24 \lambda}$, where $\eta$ is the Dedekind eta function. In particular, we have $n(n+k-1)-12 \lambda \geq 0$.

We call the inequality $n(k+n-1)-12 \lambda \geq 0$ in Theorem 1 Mason's inequality. For a vectorvalued modular form $\mathbb{F}$ whose component functions are linearly independent, a constant $\lambda_{1}+$ $\cdots+\lambda_{n}$ in the theorem does not depend on normalization. We denote $\lambda_{1}+\cdots+\lambda_{n}$ by $\lambda(\mathbb{F})$.

Let $\mathbb{F}={ }^{t}\left(f_{1}, \ldots, f_{n}\right)$ be a vector-valued modular form of weight $k$ whose component functions $f_{1}, \ldots, f_{n}$ are linearly independent. Then by Theorem 1 , there is a classical modular (not cusp) form $G$ of weight $n(d+k-1)-12 \lambda(\mathbb{F})$ such that $W(\mathbb{F})=G \eta^{24 \lambda(\mathbb{F})}$. There is the well-known identity (valence formula)

$$
\nu_{i \infty}(f)+\frac{1}{2} \nu_{i}(f)+\frac{1}{3} \nu_{e^{\pi i / 3}}(f)+\sum_{p} \nu_{p}(f)=\frac{k}{12}
$$

for any non-zero classical modular form $f$ of weight $k$, where $p\left(\neq i, e^{\pi i / 3}\right)$ runs over $\Gamma_{1} \backslash \mathbb{H}$ and $\nu_{p}(f)$ indicates the order of zero at $p$. Since $G$ is not a cusp form, we see that $G$ has zeroes in $\mathbb{H}$ if and only if $G$ has positive weight. Therefore, the modular Wronskian of $\mathbb{F}$ never vanishes in $\mathbb{H}$ if and only if $n(n+k-1)-12 \lambda(\mathbb{F})=0$ (cf. $[14,13])$.

Let $L$ be a linear differential operator of order $n$ which has the form

$$
L=\vartheta_{k}^{n}+\sum_{j=1}^{n} P_{2 j} \vartheta_{k}^{n-j},
$$

where $P_{2 j}$ is a classical modular form of weight $2 j$. Since there is no classical modular form of weight 2 , the coefficient function $P_{2}$ must be zero. It is proved in [14] that $q=0$ is a (unique) regular singular point of $L$. The linear differential equation $L(f)=0$ is called a modular linear differential equation of weight $k$ (MLDE of weight $k$ for short and see [13] for more general definition). It is also shown in [14, Theorem 4.1] that the space of solutions of a MLDE is a $\Gamma_{1}$-module with respect to the action $\left.\right|_{k}$.

Theorem 2 ([14, Theorem 4.3]). Let $\mathbb{F}={ }^{t}\left(f_{1}, \ldots, f_{n}\right)$ be a meromorphic vector-valued modular form of weight $k$ with respect to a representation $\rho: \Gamma_{1} \rightarrow G L_{n}(\mathbb{C})$. Suppose that $f_{1}, \ldots, f_{n}$ are linearly independent and normalized. Then $f_{1}, \ldots, f_{n}$ form a fundamental system of solutions of a modular linear differential equation of weight $k$ if and only if $W(\mathbb{F})$ never vanishes in $\mathbb{H}$, i.e. $n(n+k-1)-12 \lambda(\mathbb{F})=0$.

Let $\mathbb{F}={ }^{t}\left(f_{1}, \ldots, f_{n}\right)$ be a vector-valued modular form of weight $k$ whose component functions are linearly independent. We say that $\mathbb{F}$ satisfies non-zero Wronskian condition (NZWC for short) if $n(k+n-1)-12 \lambda(\mathbb{F})=0$.

\section{Preliminaries on affine vertex operator algebras and their characters}

In this section we recall the notion of affine VOAs associated with finite-dimensional simple Lie algebras and their characters as a VOA. 


\subsection{Affine vertex operator algebras}

Let $\mathfrak{g}$ be a finite-dimensional complex simple Lie algebra of rank $\ell$. Fix a Cartan subalgebra of $\mathfrak{g}$ and let $\Delta, \Delta_{+}, \Pi=\left\{\alpha_{1}, \ldots, \alpha_{\ell}\right\}$ and $\Pi^{\vee}=\left\{\alpha_{1}^{\vee}, \ldots, \alpha_{\ell}^{\vee}\right\}$ be sets of roots, positive roots, simple roots, and simple coroots, respectively. We denote by $\theta$ the highest root of $\mathfrak{g}$ and the normalized Killing form by $(\mid)$, i.e. $(\theta \mid \theta)=2$. Let $\Lambda_{i} \in \mathfrak{h}^{*}$ be a fundamental weight which is defined by $\Lambda_{i}\left(\alpha_{j}\right)=\delta_{i j}$ for every $1 \leq i \leq \ell$, and $P=\bigoplus_{i=1}^{\ell} \mathbb{Z} \Lambda_{i}$ the weight lattice. For a positive integer $k$, we call by a dominant integral weight of level $k$ an element of the set $P_{+}^{k}=\left\{\Lambda \in P \mid \Lambda\left(\alpha_{i}^{\vee}\right) \in \mathbb{Z}_{\geq 0}\right.$ for all $1 \leq i \leq \ell$ and $\left.(\theta \mid \Lambda) \leq k\right\}$. Let $\hat{\mathfrak{g}}=\mathfrak{g} \otimes \mathbb{C}\left[t, t^{-1}\right] \oplus \mathbb{C} K$ be the affine Lie algebra associated with $\mathfrak{g}$ and $L_{\mathfrak{g}, k}(\Lambda)$ the irreducible highest weight $\hat{\mathfrak{g}}$ module with highest weight $\Lambda$ and level $k$, i.e., the central element $K$ acts on $L_{\mathfrak{g}, k}(\Lambda)$ by $k$. We denote an element $x \otimes t^{n} \in \hat{\mathfrak{g}}$ by $x_{n}$. In [5] it is shown that for any positive integer $k$, the $\hat{\mathfrak{g}}$-module $L_{\mathfrak{g}, k}\left(=L_{\mathfrak{g}, k}(0)\right)$ is a simple VOA. It is known in [5, Theorem 3.1.3] that for a positive integer $k$, the list of inequivalent simple $L_{\mathfrak{g}, k}$-modules is given by $\left\{L_{\mathfrak{g}, k}(\Lambda) \mid \Lambda \in P_{+}^{k}\right\}$. Since the cardinality of $P_{+}^{k}$ is finite, the number of simple $L_{\mathfrak{g}, k}$-modules is finite. It follows form the Sugawara construction (see e.g. $[5,8]$ ) that $L_{\mathfrak{g}, k}(\Lambda)\left(\Lambda \in P_{+}^{k}\right)$ is a module of the Virasoro algebra of central charge

$$
c_{\mathfrak{g}, k}=\frac{k \operatorname{dim} \mathfrak{g}}{k+h^{\vee}},
$$

where $h^{\vee}$ is the dual Coxeter number of $\mathfrak{g}$, and that $L_{\mathfrak{g}, k}(\Lambda)=\bigoplus_{n=0}^{\infty} L_{\mathfrak{g}, k}(\Lambda)_{h_{\Lambda}+n}$ where each $L_{\mathfrak{g}, k}(\Lambda)_{h_{\Lambda}+n}$ is a finite-dimensional eigenspace of $L_{0}$ associated with an eigenvalue $h_{\Lambda}+n$. We call a constant $h_{\Lambda}$ the conformal weight of $L_{\mathfrak{g}, k}(\Lambda)$ which is known to be given by

$$
h_{\Lambda}=\frac{(\Lambda \mid \Lambda+2 \rho)}{2\left(k+h^{\vee}\right)}
$$

where $\rho$ is the Weyl vector, i.e. the sum of all fundamental weights.

Warning. In this paper we use the notation of Dynkin diagrams used in [8, Chapter 6]. The program "kac" uses the notation of Dynkin diagrams in [6].

\subsection{Characters for affine vertex operator algebras}

Let $\mathfrak{g}$ be a finite-dimensional simple Lie algebra and $k$ a positive integer. A character of a simple $L_{\mathfrak{g}, k}$-module $L_{\mathfrak{g}, k}(\Lambda)$ is a formal power series defined by

$$
\operatorname{ch}_{\Lambda}(\tau)=\operatorname{tr}_{L_{\mathfrak{g}, k}(\Lambda)} q^{L_{0}-c_{\mathfrak{g}, k} / 24}=q^{h_{\Lambda}-c_{\mathfrak{g}, k} / 24} \sum_{n=0}^{\infty} \operatorname{dim} L_{\mathfrak{g}, k}(\Lambda)_{h_{\Lambda}+n} q^{n} \quad\left(q=e^{2 \pi i \tau}\right) .
$$

Since $L_{\mathfrak{g}, k}(\Lambda)_{h_{\Lambda}}$ is a finite-dimensional irreducible $\mathfrak{g}$-module of highest weight $\Lambda$ as shown in [5], the leading coefficient of the character $\operatorname{ch}_{\Lambda}$ (the dimension of $L_{\mathfrak{g}, k}(\Lambda)_{h_{\Lambda}}$ ) is given by Weyl's dimension formula (see [7, $§ 24.2$, Corollary]). It is shown in [9] and [19] that $\operatorname{ch}_{\Lambda}(\tau)$ absolutely and uniformly converses on the complex upper half-plane and then defines a holomorphic function on $\mathbb{H}$. We denote by $\mathcal{X}_{\mathfrak{g}, k}$ the vector space of characters of all simple $L_{\mathfrak{g}, k}-$ modules. 


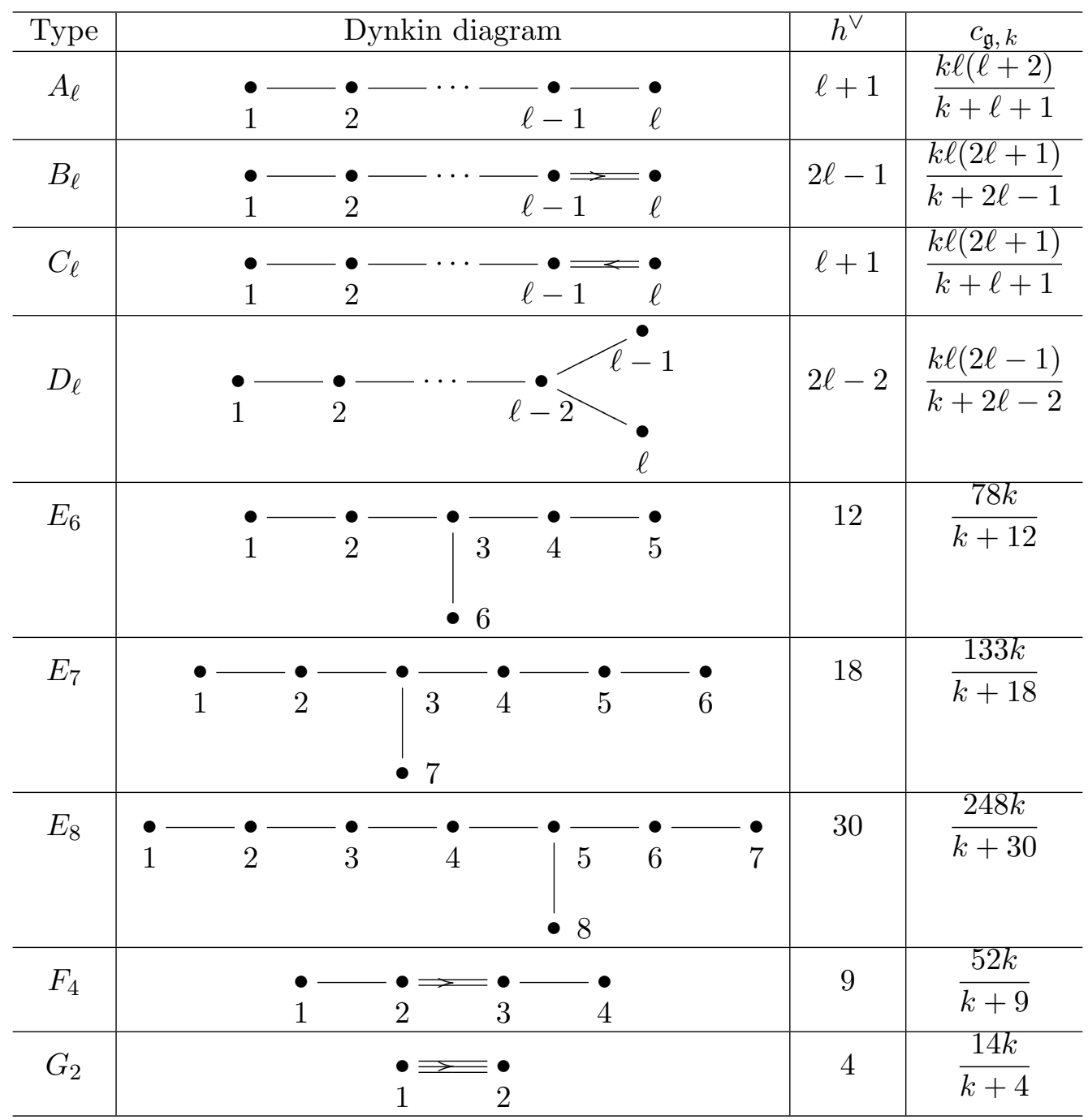

Table 1: Dynkin diagrams, dual Coxeter numbers of simple Lie algebras and central charges 
Theorem $3([9,19])$. Let $\mathfrak{g}$ be a finite-dimensional simple Lie algebra and $k$ a positive integer. Then the space $\mathcal{X}_{\mathfrak{g}, k}$ is a module of $S L_{2}(\mathbb{Z})$ by the slash $\left.\right|_{0}$-action and $\operatorname{dim} \mathcal{X}_{\mathfrak{g}, k} \leq\left|P_{+}^{k}\right|$. In particular, the column vector-valued function consisting of characters of all inequivalent simple $L_{\mathfrak{g}, k}$-modules is a meromorphic vector-valued modular form of weight 0 .

Remark. Let $\left\{f_{1}, \ldots, f_{n}\right\}$ be a normalized basis of $\mathcal{X}_{\mathfrak{g}, k}$. If the vector-valued modular form ${ }^{t}\left(f_{1}, \ldots, f_{n}\right)$ satisfies non-zero Wronskian condition, we say that " $L_{\mathfrak{g}, k}$ satisfies nonzero Wronskian condition".

By the very definition of the characters, we have the following lemma.

Lemma 4. The characters with mutually distinct conformal weights are linearly independent.

We identify the index set $\{1,2, \ldots, \ell\}$ with the set of vertices of the Dynkin diagram associated with $\mathfrak{g}$ in the usual way (see Table 1 ). Let $\bar{\sigma}$ be an automorphism of the Dynkin diagram in Table 1. Then we can extend $\bar{\sigma}$ to a Lie algebra automorphism $\sigma$ : For Chevalley generators $e_{1}, \ldots, e_{\ell}, \alpha_{1}^{\vee}, \ldots, \alpha_{\ell}^{\vee}, f_{1}, \ldots, f_{\ell}$, the automorphism $\sigma$ of $\mathfrak{g}$ is defined by $\sigma\left(e_{i}\right)=e_{\bar{\sigma}(i)}$, $\sigma\left(f_{i}\right)=f_{\bar{\sigma}(i)}, \sigma\left(\alpha_{i}^{\vee}\right)=\alpha_{\bar{\sigma}(i)}^{\vee}$. Such an automorphism $\sigma$ is called the diagram automorphism of $\mathfrak{g}$.

Proposition $5([7, \S 12.2])$. The groups of automorphisms of Dynkin diagrams are trivial except for $A_{\ell}, D_{\ell}$ and $E_{6}$.

(1) The group of automorphisms of the Dynkin diagram $A_{\ell}$ is $\mathbb{Z}_{2}$. The non-trivial automorphism $\bar{\sigma}$ is given by $\bar{\sigma}(i)=\ell+1-i$ for each $1 \leq i \leq \ell$, where $i$ is a vertex of the Dynkin diagram.

(2) The group of automorphisms of the Dynkin diagram $D_{\ell}(\ell>4)$ is the permutation group $\mathbb{Z}_{2}$ of the set of vertices $\ell-1$ and $\ell$.

(3) The group of automorphisms of the Dynkin diagram $D_{4}$ is isomorphic to the symmetric group $S_{3}$ of degree 3 which permutes vertices 1,3, 4 of the Dynkin diagram.

(4) The group of automorphisms of the Dynkin diagram $E_{6}$ is isomorphic to $\mathbb{Z}_{2}$. The nontrivial automorphism $\bar{\sigma}$ is given by $\bar{\sigma}(i)=6-i$ for each $1 \leq i \leq 5$.

Let $\sigma$ be a diagram automorphism of $\mathfrak{g}$. Then we can define an automorphism of $\hat{\mathfrak{g}}$ by $\sigma\left(a \otimes t^{n}\right)=\sigma(a) \otimes t^{n}$ and $\sigma(K)=K$. Each diagram automorphism $\sigma$ defines a $\hat{\mathfrak{g}}-$ module $L_{\mathfrak{g}, k}(\Lambda)^{\sigma}$ by $\left(a \otimes t^{n}\right) \cdot v=\left(\sigma(a) \otimes t^{n}\right) v$ for every $v \in L_{\mathfrak{g}, k}(\Lambda)$ and $a \otimes t^{n} \in \hat{\mathfrak{g}}$. Write $\Lambda \in P_{+}^{k}$ as $\Lambda=\sum_{i=1}^{\ell} m_{i} \Lambda_{i}$ where each $m_{i}$ is a non-negative integer. Since $\left(\alpha_{i}^{\vee}\right)_{0} \cdot v_{\Lambda}=$ $\sigma\left(\alpha_{i}^{\vee}\right)_{0} v_{\Lambda}=\Lambda\left(\alpha_{\sigma(i)}^{\vee}\right) v_{\Lambda}$, the highest weight vector $v_{\Lambda}$ of $L_{\mathfrak{g}, k}(\Lambda)$ is also a highest weight vector of $L_{\mathfrak{g}, k}(\Lambda)^{\sigma}$ with highest weight $\sum_{i=1}^{\ell} m_{\bar{\sigma}(i)} \Lambda_{i}$, which is denoted by $\Lambda^{\sigma}$. Therefore, it follows from Proposition 5 that $\Lambda \in P_{+}^{k}$ if and only if $\Lambda^{\sigma} \in P_{+}^{k}$.

Let $\sigma$ be a diagram automorphism of a finite-dimensional simple Lie algebra $\mathfrak{g}$. Then $\sigma$ can be extended to an automorphism of the VOA $L_{\mathfrak{g}, k}$. Since, by definition, any automorphism of a VOA preserves the Virasoro element, the characters $\operatorname{ch}_{\Lambda}(\tau)$ and $\operatorname{ch}_{\Lambda^{\sigma}}(\tau)$ must coincide. Motivated by this fact we introduce an equivalence relation $\sim$ on $P_{+}^{k} ; \Lambda \sim \Lambda^{\prime}$ if and only if there is a diagram automorphism $\sigma$ such that $\Lambda^{\prime}=\Lambda^{\sigma}$, and set $\widetilde{P}_{+}^{k}=P_{+}^{k} / \sim$. By Lemma 4 , we have: 
Proposition 6. Let $k$ be a positive integer and $L_{\mathfrak{g}, k}$ an affine vertex operator algebra associated with a finite-dimensional simple Lie algebra $\mathfrak{g}$. Suppose that conformal weights of simple modules $L_{\mathfrak{g}, k}(\Lambda)$ for $\Lambda \in \widetilde{P}_{+}^{k}$ are mutually distinct. Then the dimension of the space of characters of $L_{\mathfrak{g}, k}$ is given by $\left|\widetilde{P}_{+}^{k}\right|$.

\section{Characters and non-zero Wronskian condition for affine vertex operator algebras}

In this section we show that a basis of the space of characters of an affine VOA whose dimension of the space of characters is $2,3,4$, or 5 forms a fundamental system of solutions of a MLDE of weight 0 .

Let $\mathfrak{g}$ be a finite-dimensional simple Lie algebra of type $X_{\ell}$. We denote by $L_{X_{\ell}, k}(\Lambda)$ the irreducible highest weight $\hat{\mathfrak{g}}$-module with highest weight $\Lambda$. One of the main results in this paper is:

Theorem 7. An affine vertex operator algebra of positive integral level whose dimension of the space of characters is not exceeding 5 is isomorphic to one of $L_{\mathfrak{g}, 1}\left(\mathfrak{g}=A_{\ell}(1 \leq \ell \leq 8)\right.$, $\left.B_{\ell}(\ell \geq 2), C_{\ell}(2 \leq \ell \leq 4), D_{\ell}(\ell \geq 4), E_{6}, E_{7}, E_{8}, G_{2}, F_{4}\right)$, and $L_{\mathfrak{g}, 2}\left(\mathfrak{g}=A_{1}, A_{2}, D_{4}\right.$, $\left.E_{8}, F_{4}, G_{2}\right)$, and $L_{\mathfrak{g}, 3}\left(\mathfrak{g}=A_{1}, E_{8}\right)$, and $L_{A_{1}, 4}$. These affine vertex operator algebras satisfy non-zero Wronskian condition except for $L_{E_{8}, 1}$.

Remark. Affine VOAs with $\operatorname{dim} \mathcal{X}_{\mathfrak{g}, k}=3$ are $L_{\mathfrak{g}, 1}\left(\mathfrak{g}=A_{3}, A_{4}, B_{\ell}(\ell \geq 3), C_{2}, D_{\ell}(\ell \geq 5)\right)$, and $L_{\mathfrak{g}, 2}\left(\mathfrak{g}=A_{1}, E_{8}\right)$ (see Theorems 9, 11-13 and Table 2).

The remaining of this section is devoted to a proof of this theorem on a case-by-case basis. First of all, the next lemma is applied to each case, which immediately follows from (5), Lemma 4 and the fact $P_{+}^{k} \subset P_{+}^{k+1}$.

Lemma 8. Let $S \subset P_{+}^{k}$ be a subset. Then conformal weights of $L_{\mathfrak{g}, k+1}(\Lambda)$ for all $\Lambda \in S$ are mutually distinct if and only if conformal weights of $L_{\mathfrak{g}, k}(\Lambda)(\Lambda \in S)$ are mutually distinct.

We start with type $A$.

(1) Type $A$. The system of fundamental weights and the highest root of $A_{\ell}$ are written by using elements of $\mathbb{R}^{\ell+1}$ as

$$
\Lambda_{i}=\frac{1}{\ell+1}(\overbrace{\ell+1-i, \ldots, \ell+1-i}^{i}, \overbrace{-i, \ldots,-i}^{\ell+1-i}), \quad \theta=(1,0, \ldots, 0,1)
$$

for $1 \leq i \leq \ell$. Then it is not hard to show that $\left(\Lambda_{i} \mid \Lambda_{j}\right)=j(\ell+1-i) /(\ell+1)(1 \leq j \leq i \leq \ell)$ and $\left(\Lambda_{i} \mid \rho\right)=i(\ell+1-i) / 2(1 \leq i \leq \ell)$. The set of inequivalent simple modules of $L_{A_{\ell}, k}$ is given by

$$
\left\{L_{A_{\ell}, k}\left(\sum_{i=1}^{\ell} m_{i} \Lambda_{i}\right) \mid m_{i} \in \mathbb{Z}_{\geq 0}, m_{1}+m_{2}+\cdots+m_{\ell} \leq k\right\} .
$$


Theorem 9. Let $\mathcal{X}_{A_{\ell}, k}$ be the space of characters of $L_{A_{\ell}, k}$ and $k, \ell \geq 1$ integers. Then we have $\operatorname{dim} \mathcal{X}_{A_{\ell}, 1}=\lfloor(\ell+1) / 2\rfloor+1, \operatorname{dim} \mathcal{X}_{A_{1}, k}=k+1$ and $\operatorname{dim} \mathcal{X}_{A_{2}, 2}=4$. Further $L_{A_{\ell}, 1}$, $L_{A_{1}, k}$ and $L_{A_{2}, 2}$ satisfy non-zero Wronskian condition.

Proof. Since the list of inequivalent simple $L_{A_{1}, k}$-modules is $\left\{L_{A_{1}, k}\left(j \Lambda_{1}\right) \mid 0 \leq j \leq k\right\}$, the conformal weights of $L_{A_{1}, k}\left(j \Lambda_{1}\right)$ for $0 \leq j \leq k$ are $j(j+2) / 4(k+2)$, which are mutually distinct. Therefore we have $\operatorname{dim} \mathcal{X}_{A_{1}, k}=k+1$. Non-zero Wronskian condition immediately follows from

$$
\sum_{j=0}^{k}\left(\frac{j(j+4)}{4(k+2)}-\frac{3 k}{24(k+2)}\right)=\frac{1}{12} k(k+1) .
$$

We next prove that $\operatorname{dim} \mathcal{X}_{A_{\ell}, 1}=\lfloor(\ell+1) / 2\rfloor+1$. Since the list of inequivalent simple $L_{A_{\ell}, 1}$-modules is $L_{A_{\ell}, 1}, L_{A_{\ell}, 1}\left(\Lambda_{i}\right)(1 \leq i \leq \ell)$, it follows from Proposition 5 that $\operatorname{dim} \mathcal{X}_{A_{\ell}, 1}$ is at most $\lfloor(\ell+1) / 2\rfloor+1$. Conformal weights of $L_{A_{\ell}, 1}\left(\Lambda_{i}\right)(1 \leq i \leq\lfloor(\ell+1) / 2\rfloor)$ are given by $i(\ell+1-i) / 2(\ell+1)$. Because $i(\ell+1-i) / 2(\ell+1)$ and $j(\ell+1-j) / 2(\ell+1)$ are mutually distinct if and only if $i=j$ or $i=\ell+1-j$, which shows $\operatorname{dim} \mathcal{X}_{A_{\ell}, 1}=\lfloor(\ell+1) / 2\rfloor+1$.

Finally we prove $\operatorname{dim} \mathcal{X}_{A_{2}, 2}=4$. The set $\widetilde{P}_{+}^{2}$ consists of highest weights $0, \Lambda_{1}, \Lambda_{1}+\Lambda_{2}$, $2 \Lambda_{1}$ whose conformal weights are $0,4 / 15,2 / 3,3 / 5$, respectively. Now, non-zero Wronskian condition follows.

Proposition 10. For each integer $k \geq 2$ and $\ell \geq 1$, we have $\operatorname{dim} \mathcal{X}_{A_{\ell}, k} \geq k+\ell$.

Proof. It is obvious that $\widetilde{P}_{+}^{k}$ contains highest weights $\Lambda_{1}+\Lambda_{i}(2 \leq i \leq \ell), j \Lambda_{1}(0 \leq j \leq k)$ whose conformal weights are $\left(2 \ell^{2}+6 \ell-i^{2}-2 i+3\right) / 2(\ell+1)(k+\ell+1)(2 \leq i \leq \ell)$, $j \ell(\ell+j+1) / 2(\ell+1)(k+\ell+1)$. The conformal weights of $L_{A_{\ell}, k}\left(\Lambda_{1}+\Lambda_{i}\right)(2 \leq i \leq \ell)$ are mutually distinct and ones of $L_{A_{\ell}, k}\left(j \Lambda_{1}\right)(0 \leq j \leq k)$ are also mutually distinct. Suppose that $j \ell(\ell+j+1) / 2(\ell+1)(k+\ell+1)=\left(2 \ell^{2}+6 \ell-i^{2}-2 i+3\right) / 2(\ell+1)(k+\ell+1)$. Then we have $i=1$ and $j=2$, which contradicts to the assumption $2 \leq i \leq \ell$ and $0 \leq j \leq k$. Therefore the conformal weights of $L_{A_{\ell}, k}\left(\Lambda_{1}+\Lambda_{i}\right)$ and $L_{A_{\ell}, k}\left(j \Lambda_{1}\right)$ are mutually distinct, which shows that $\operatorname{dim} \mathcal{X}_{A_{\ell}, 2} \geq(k+1)+(\ell-1)=k+\ell$.

Proof of Theorem 7 for type $A$. By Theorem 9, Proposition 10 and Lemma 8, it suffices to prove that $\operatorname{dim} \mathcal{X}_{A_{3}, 2} \geq 6$ and $\operatorname{dim} \mathcal{X}_{A_{2}, 3} \geq 6$. By direct calculation, we see that the number of mutually distinct conformal weights of simple $L_{A_{3}, 2}$-modules and simple $L_{A_{2}, 3}$-modules are 7 and 6 , respectively. Therefore it follows that $\operatorname{dim} \mathcal{X}_{A_{\ell}, k} \leq 5$ if and only if $(k, \ell)=(1, \ell)$ for $1 \leq \ell \leq 8,(k, \ell)=(k, 1)$ for $1 \leq k \leq 4$, or $(k, \ell)=(2,2)$.

(2) Type $B$. The set of fundamental weights $\Lambda_{i}$ for the finite-dimensional simple Lie algebra of type $B_{\ell}(\ell \geq 3)$ is written by using elements of $\mathbb{R}^{\ell}$ as

$$
\Lambda_{i}=\left\{\begin{array}{ll}
(\underbrace{1, \ldots, 1}_{i}, 0, \ldots, 0) & 1 \leq i \leq \ell-1, \\
\frac{1}{2}(1, \ldots, 1) & i=\ell,
\end{array} \quad \rho=\frac{1}{2}(2 \ell-1,2 \ell-3, \ldots, 3,1) .\right.
$$


Then we see that

$$
\begin{aligned}
& \left(\Lambda_{i} \mid \Lambda_{j}\right)=i \quad(1 \leq i \leq j \leq \ell-1), \quad\left(\Lambda_{i} \mid \Lambda_{\ell}\right)=\frac{i}{2} \quad(1 \leq i \leq \ell-1), \\
& \left(\Lambda_{\ell} \mid \Lambda_{\ell}\right)=\frac{\ell}{4}, \quad\left(\Lambda_{i} \mid \rho\right)= \begin{cases}\frac{1}{2} i(2 \ell-i) & 1 \leq i \leq \ell-1, \\
\frac{1}{4} \ell^{2} & i=\ell .\end{cases}
\end{aligned}
$$

and the highest root is given by $\theta=(1,1,0, \ldots, 0)$. Then the list of inequivalent simple $L_{B_{\ell}, k}$-modules is

$$
\left\{L_{B_{\ell}, k}\left(\sum_{i=1}^{\ell} m_{i} \Lambda_{i}\right) \mid m_{i} \in \mathbb{Z}_{\geq 0}, m_{1}+2 \sum_{i=2}^{\ell-1} m_{i}+m_{\ell} \leq k\right\} .
$$

Theorem 11. Let $\mathcal{X}_{B_{\ell}, k}$ be the space of characters of $L_{B_{\ell}, k}$ and $k \geq 2, \ell \geq 3$ integers. Then $\operatorname{dim} \mathcal{X}_{B_{\ell}, 1}=3$ and $\operatorname{dim} \mathcal{X}_{B_{\ell}, k} \geq \ell+1$. Further $L_{B_{\ell}, 1}$ satisfies non-zero Wronskian condition.

Proof. The highest weights of simple $L_{B_{\ell}, 1}$-modules are $0, \Lambda_{1}, \Lambda_{\ell}$. By (5) and (7), the conformal weights of simple modules are $0,1 / 2,(2 \ell+1) / 16$, and then by Lemma 4 , we have $\operatorname{dim} \mathcal{X}_{B_{\ell}, 1}=3$. Since the sum of all conformal weights is $(2 \ell+9) / 16$ and the central charge is $(2 \ell+1) / 2$, non-zero Wronskian condition simply holds.

The set $P_{+}^{k}$ for $k>1$ contains highest weights $\Lambda_{1}, \Lambda_{2}, \ldots, \Lambda_{\ell-1}, 2 \Lambda_{\ell}$ whose conformal weights are $i(2 \ell+1-i) / 2(k+2 \ell-1)(1 \leq i \leq \ell-1)$ and $\ell(\ell+1) / 2(k+2 \ell-1)$. It is obvious that $i(2 \ell+1-i)(1 \leq i \leq \ell-1)$ are mutually distinct and that $i(2 \ell+1-i)$ is equal to $\ell(\ell+1)$ if and only if $i=\ell$ or $i=\ell+1$, which is impossible since $1 \leq i \leq \ell-1$. Therefore conformal weights of simple modules with highest weights $0, \Lambda_{1}, \ldots, \Lambda_{\ell-1}$ and $2 \Lambda_{\ell}$ are mutually distinct, which shows that $\operatorname{dim} \mathcal{X}_{B_{\ell}, k} \geq \ell+1$ by Lemma 8 .

Proof of Theorem 7 for type $B$. By Lemma 8 and Theorem 11, it suffices to prove that $\operatorname{dim} \mathcal{X}_{B_{3}, 2} \geq 6$ and $\operatorname{dim} \mathcal{X}_{B_{4}, 2} \geq 6$. Since highest weights of inequivalent simple $L_{B_{3}, 2^{-}}$ modules are $0, \Lambda_{1}, \Lambda_{2}, \Lambda_{3}, 2 \Lambda_{1}, 2 \Lambda_{3}, \Lambda_{1}+\Lambda_{3}$ and their conformal weights are $0,3 / 7,5 / 7$, $3 / 8,1,6 / 7,7 / 8$, respectively, thus we have $\operatorname{dim} \mathcal{X}_{B_{3}, 2}=6$. It follows that highest weights of inequivalent simple $L_{B_{4}, 2}$ are $0, \Lambda_{1}, \Lambda_{2}, \Lambda_{3}, \Lambda_{4}, 2 \Lambda_{1}, 2 \Lambda_{4}, \Lambda_{1}+\Lambda_{4}$ and their conformal weights are $0,4 / 9,7 / 9,1,1 / 2,1,10 / 9,1$, respectively, which implies that $\operatorname{dim} \mathcal{X}_{B_{4}, 2} \geq 6$. Therefore $\operatorname{dim} \mathcal{X}_{B_{\ell}, k} \leq 5$ if and only if $k=1$.

(3) Type $C$. The system of fundamental weights and the Weyl vector of finite-dimensional simple Lie algebra of type $C$ is written by using elements of $\mathbb{R}^{\ell}$ as

$$
\Lambda_{i}=\frac{1}{\sqrt{2}}(\overbrace{1, \ldots, 1}^{i}, \overbrace{0, \ldots, 0}^{\ell-i}), \quad \rho=\frac{1}{\sqrt{2}}(\ell, \ell-1, \ldots, 2,1)
$$

for $1 \leq i \leq \ell$. Therefore it follows that

$$
\left(\Lambda_{i} \mid \Lambda_{j}\right)=\frac{1}{2} i \quad(1 \leq i \leq j \leq \ell), \quad\left(\Lambda_{i} \mid \rho\right)=\frac{1}{4} i(2 \ell+1-i) .
$$

The highest root is given by $\theta=(\sqrt{2}, 0, \ldots, 0)$ and the list of inequivalent simple $L_{C_{\ell}, k^{-}}$ modules is

$$
\left\{L_{C_{\ell}, k}\left(\sum_{i=1}^{\ell} m_{i} \Lambda_{i}\right) \mid m_{i} \in \mathbb{Z}_{\geq 0}, \sum_{i=1}^{\ell} m_{i} \leq k\right\} .
$$


Theorem 12. Let $\mathcal{X}_{C_{\ell}, k}$ be the space of characters of $L_{C_{\ell}, k}$ and $\ell \geq 2$ an integer. Then $\operatorname{dim} \mathcal{X}_{C_{\ell}, 1}=\ell+1$ and $L_{C_{\ell}, 1}$ satisfies non-zero Wronskian condition.

Proof. The highest weights of simple $L_{C_{\ell}, 1}$-modules are $0, \Lambda_{i}(1 \leq i \leq \ell)$ whose conformal weights are respectively given by $0, i(2 \ell+2-i) / 4(\ell+2)$ by $(5)$ and (8). Then $i(2 \ell+2-$ $i) / 4(\ell+2)=j(2 \ell+2-j) / 4(\ell+2)$ if and only if $i=j$ or $j=2 \ell+2-i$, which shows that conformal weights are mutually distinct. Hence by Lemma 4 , we have $\operatorname{dim} \mathcal{X}_{C_{\ell}, 1}=\ell+1$. Non-zero Wronskian condition simply follows.

A lower bound of $\operatorname{dim} \mathcal{X}_{C_{\ell}, k}$ follows from Lemma 8.

Corollary. $\operatorname{dim} \mathcal{X}_{C_{\ell}, k} \geq \ell+1$ for each integer $\ell \geq 2$ and $k \geq 1$.

Proof of Theorem 7 for type $C$. By Lemma 8, it suffices to prove that $\operatorname{dim} \mathcal{X}_{C_{\ell}, 2} \geq 6$ for $\ell \geq 2$. The elements $0, \Lambda_{1}, \Lambda_{2}, 2 \Lambda_{1}, 2 \Lambda_{2}, \Lambda_{1}+\Lambda_{2}$ are highest weights of simple $L_{C_{\ell}, 2}$-modules and their conformal weights are $0,(2 \ell+1) / 4(\ell+3), \ell /(\ell+3),(\ell+1) /(\ell+3),(2 \ell+1) /(\ell+3)$, $(6 \ell+3) / 4(\ell+3)$, respectively. It is obvious that these conformal weights are mutually distinct, which shows that $\operatorname{dim} \mathcal{X}_{C_{\ell}, 2} \geq 6$.

(4) Type $D$. Let $\Lambda_{i}(1 \leq i \leq \ell)$ be the system of fundamental weights of the finitedimensional simple Lie algebra of type $D_{\ell}(\ell \geq 4)$. Then fundamental weights and the

Weyl vector are equated with elements of $\mathbb{R}^{\ell}: \Lambda_{i}=(\overbrace{1, \ldots, 1}^{i}, \overbrace{0, \ldots, 0}^{\ell-i})(1 \leq i \leq \ell-2)$, $\Lambda_{\ell-1}=(1 / 2, \ldots, 1 / 2,-1 / 2), \Lambda_{\ell}=(1 / 2, \ldots, 1 / 2)$ and $\rho=(\ell-1, \ell-2, \ldots, 2,1,0)$. Then we have

$$
\begin{aligned}
& \left(\Lambda_{i} \mid \Lambda_{j}\right)=i \quad(1 \leq i \leq j \leq \ell-2), \quad\left(\Lambda_{i} \mid \Lambda_{\ell-1}\right)=\left(\Lambda_{i} \mid \Lambda_{\ell}\right)=\frac{i}{2} \quad(1 \leq i \leq \ell-2), \\
& \left(\Lambda_{\ell-1} \mid \Lambda_{\ell-1}\right)=\left(\Lambda_{\ell} \mid \Lambda_{\ell}\right)=\frac{\ell}{4}, \quad\left(\Lambda_{\ell-1} \mid \Lambda_{\ell}\right)=\frac{\ell-2}{4}, \\
& \left(\Lambda_{i} \mid \rho\right)= \begin{cases}\frac{1}{2} i(2 \ell-i-1) & 1 \leq i \leq \ell-2, \\
\frac{1}{4} \ell(\ell-1) & i=\ell-1, \ell .\end{cases}
\end{aligned}
$$

Since the highest root is $\theta=(1,1,0, \ldots, 0)$, the list of inequivalent simple $L_{D_{\ell}, k}$-modules is given by

$$
\left\{L_{D_{\ell}, k}\left(\sum_{i=1}^{\ell} m_{i} \Lambda_{i}\right) \mid m_{i} \in \mathbb{Z}_{\geq 0}, m_{1}+2 \sum_{i=2}^{\ell-2} m_{i}+m_{\ell-1}+m_{\ell} \leq k\right\} .
$$

Theorem 13. Let $\mathcal{X}_{D_{\ell}, k}$ be the space of characters of $L_{D_{\ell}, k}$. For each positive integer $\ell \geq 5$, $\operatorname{dim} \mathcal{X}_{D_{4}, 1}=2, \operatorname{dim} \mathcal{X}_{D_{\ell}, 1}=3$ and $\operatorname{dim} \mathcal{X}_{D_{4}, 2}=5$. Further $L_{D_{\ell}, 1}(\ell \geq 4)$ and $L_{D_{4}, 2}$ satisfy non-zero Wronskian condition.

Proof. The highest weights of simple $L_{D_{\ell}, 1}$-modules are $0, \Lambda_{1}, \Lambda_{\ell-1}$ and $\Lambda_{\ell}$. By Proposition 5, the characters $\operatorname{ch}_{\Lambda_{\ell-1}}$ and $\operatorname{ch}_{\Lambda_{\ell}}$ coincide, and for $\ell=4$ the character $\operatorname{ch}_{\Lambda_{1}}$ coincides with $\operatorname{ch}_{\Lambda_{3}}$ and $\operatorname{ch}_{\Lambda_{4}}$. It follows from (5) and (9) that all conformal weights are given by 0 , $1 / 2, \ell / 8$. By Proposition 6 we have $\operatorname{dim} \mathcal{X}_{D_{\ell}, 1}=3(\ell>4)$ and $\operatorname{dim} \mathcal{X}_{D_{4}, 1}=2$. The elements of $\widetilde{P}_{+}^{2}$ for $L_{D_{4}, 2}$ are $0, \Lambda_{1}, 2 \Lambda_{1}, \Lambda_{1}+\Lambda_{3}, \Lambda_{2}$ whose conformal weights are $0,7 / 16,1,15 / 16$, and $3 / 4$, respectively, which shows that $\operatorname{dim} L_{D_{4}, 2}=5$. Non-zero Wronskian condition is obvious since the central charges of $L_{D_{\ell}, 1}$ and $L_{D_{4}, 2}$ are $\ell$ and 7 , respectively. 


\begin{tabular}{c|c|c|c|c|c|c|c|c|c|c|c|c|c|c}
\hline Type $\mathfrak{g}$ & $E_{6}$ & $E_{6}$ & $E_{7}$ & $E_{7}$ & $E_{8}$ & $E_{8}$ & $E_{8}$ & $E_{8}$ & $F_{4}$ & $F_{4}$ & $F_{4}$ & $G_{2}$ & $G_{2}$ & $G_{2}$ \\
\hline Level $k$ & 1 & 2 & 1 & 2 & 1 & 2 & 3 & 4 & 1 & 2 & 3 & 1 & 2 & 3 \\
\hline $\operatorname{dim} \mathcal{X}_{\mathfrak{g}, k}$ & 2 & 6 & 2 & 6 & 1 & 3 & 5 & 10 & 2 & 5 & 9 & 2 & 4 & 6 \\
\hline
\end{tabular}

Table 2: $\operatorname{dim} \mathcal{X}_{\mathfrak{g}, k}$ for exceptional types

Proposition 14. We have $\operatorname{dim} \mathcal{X}_{D_{\ell}, k} \geq \ell+1$ for each integer $\ell \geq 4$ and $k \geq 2$.

Proof. The set $P_{+}^{k}$ contains highest weights $0, \Lambda_{i}(1 \leq i \leq \ell-2), 2 \Lambda_{\ell}, \Lambda_{\ell-1}+\Lambda_{\ell}$ whose conformal weights are respectively given by $0, i(2 \ell-i) / 2(k+2 \ell-2)(1 \leq i \leq \ell-2)$, $\ell^{2} /(k+2 \ell-2)$ and $\left(\ell^{2}-1\right) /(k+2 \ell-2)$. Since these conformal weights are mutually distinct, we have $\operatorname{dim} \mathcal{X}_{D_{\ell}, k} \geq \ell+1$.

Proof of Theorem 7 for type D. By Lemma 8, Theorem 13 and Proposition 14 it suffices to prove that $\operatorname{dim} \mathcal{X}_{D_{4}, 3} \geq 6$. We see that there are simple $L_{D_{4}, 3}$-modules whose conformal weights are $0,7 / 18,8 / 9,3 / 2,5 / 6,25 / 18,2 / 3,7 / 6$ and $4 / 3$. Thus it follows that $\operatorname{dim} \mathcal{X}_{D_{4}, 3} \geq 9$.

(5) Exceptional types. The list of fundamental weights of finite-dimensional simple Lie algebras of exceptional types is found in [7, pp. 69]. By (5) and Lemma 4, we obtain Table 2 (for $E_{6}$, we also use Propositions 5 and 6). Affine VOAs in Table 2 with $2 \leq \operatorname{dim} \mathcal{X}_{\mathfrak{g}, k} \leq 5$ satisfy non-zero Wronskian condition.

\section{Tables of dimensions of spaces of characters of simple modules}

In this section we give lists of affine VOAs of positive integral levels, which have simple modules not exceeding 20. Each list includes information of central charges, dimensions of the spaces of characters and non-zero Wronskian condition.

Remark. Let $C_{\mathfrak{g}, k}$ be the set of inequivalent simple modules of an affine VOA of level $k$ associated with the finite-dimensional simple Lie algebra $\mathfrak{g}$ such that $\sharp C_{\mathfrak{g}, k} \leq 20$. There are 117 and two discrete infinite series of affine VOAs with this property.

(1) Suppose that $L_{\mathfrak{g}, k}$ is neither $L_{B_{4}, 2}$ nor $L_{B_{4}, 3}$. Then the characters in $\mathcal{X}_{\mathfrak{g}, k}$ with same conformal weights are linearly independent if they are distinct each other.

(2) If $L_{\mathfrak{g}, k}$ does not satisfy non-zero Wronskian condition, then there are two conformal weights that differ by an integer. However, the converse is not true. In fact, conformal weights of simple $L_{D_{8}, 1}$-modules are $0,1 / 2,1$, although $L_{D_{8}, 1}$ satisfies non-zero Wronskian condition.

We give tables of dimensions of $\mathcal{X}_{\mathfrak{g}, k}$ and explain methods which we used to determine $\operatorname{dim} \mathcal{X}_{\mathfrak{g}, k}$ as well as non-zero Wronskian condition. Each table consists of data of types of Lie algebras, levels, central charges, dimensions of the spaces of characters and non-zero Wronskian condition (we write $\bigcirc$ if $L_{\mathfrak{g}, k}$ satisfies non-zero Wronskian condition and $\times$ otherwise). Furthermore, we indicate a method by which we determine $\operatorname{dim} \mathcal{X}_{\mathfrak{g}, k}$ in the 5 th row. Each of symbols $-, \bigcirc, \diamond$ means that we have determined $\operatorname{dim} \mathcal{X}_{\mathfrak{g}, k}$ by using the the fact $L_{\mathfrak{g}, k}$ 
has mutually different conformal weights, diagram automorphisms (Propositions 5 and 6), $S$-matrices (which are explained in the next section).

We start with affine VOAs which have 4 simple modules.

1) 4 simple modules. There are 5 affine VOAs and a (discrete) series of affine VOAs which have 4 simple modules; $L_{A_{1}, 3}, L_{A_{3}, 1}, L_{C_{3}, 1}, L_{D_{4}, 1}, L_{D_{\ell}, 1}(\ell \geq 5)$ and $L_{G_{2}, 2}$.

\begin{tabular}{c|c|c|c|c|c|c}
\hline Information & $A_{1}$ & $A_{3}$ & $C_{3}$ & $D_{4}$ & $D_{\ell}(\ell \geq 5)$ & $G_{2}$ \\
\hline \hline Level $k$ & 3 & 1 & 1 & 1 & 1 & 2 \\
\hline Central charge & $9 / 5$ & 3 & $21 / 5$ & 4 & $\ell$ & $14 / 3$ \\
\hline $\operatorname{dim} \mathcal{X}_{\mathfrak{g}, k}$ & 4 & 3 & 4 & 2 & 3 & 4 \\
\hline Method & - & $\bigcirc$ & - & $\bigcirc$ & $\bigcirc$ & - \\
\hline NZWC & $\circ$ & $\circ$ & $\bigcirc$ & $\bigcirc$ & $\bigcirc$ & $\circ$ \\
\hline
\end{tabular}

Table 3: 4 simple modules

2) 5 simple modules. There are 5 affine VOAs whose numbers of inequivalent simple modules are $5 ; L_{A_{1}, 4}, L_{A_{4}, 1}, L_{C_{4}, 1}, L_{E_{8}, 3}$ and $L_{F_{4}, 2}$.

\begin{tabular}{|c|c|c|c|c|c|}
\hline Information & $A_{1}$ & $A_{4}$ & $C_{4}$ & $E_{8}$ & $F_{4}$ \\
\hline$\overline{\text { Level } k}$ & $\overline{4}$ & $\overline{1} 1$ & $\overline{1} 1$ & $\overline{3}$ & 2 \\
\hline Central charge & 2 & 4 & 6 & $248 / 11$ & $104 / 11$ \\
\hline $\operatorname{dim} \mathcal{X}_{\mathfrak{g}, k}$ & 5 & 3 & 5 & 5 & 5 \\
\hline Method & - & 0 & - & - & - \\
\hline NZWC & 0 & 0 & 0 & 0 & 0 \\
\hline
\end{tabular}

Table 4: 5 simple modules

3) 6 simple modules. There are 7 affine VOAs whose numbers of inequivalent simple modules are $6 ; L_{A_{1}, 5}, L_{A_{2}, 2}, L_{A_{5}, 1}, L_{C_{2}, 2}, L_{C_{5}, 1}, L_{E_{7}, 3}$ and $L_{G_{2}, 2}$.

4) 7 simple modules. There are 4 affine VOAs whose numbers of inequivalent simple modules are $7 ; L_{A_{1}, 6}, L_{A_{6}, 1}, L_{B_{3}, 2}$ and $L_{C_{6}, 1}$.

5) 8 simple modules. There are 4 affine VOAs whose numbers of inequivalent simple modules are 8; $L_{A_{1}, 7}, L_{A_{7}, 1}, L_{B_{4}, 2}$ and $L_{C_{7}, 1}$. The dimension $\mathcal{X}_{B_{4}, 2}$ is determined in Proposition 16 in $\S 5$.

6) 9 simple modules. There are 7 affine VOAs whose numbers of inequivalent simple modules are $9 ; L_{A_{1}, 8}, L_{A_{8}, 1}, L_{B_{5}, 2}, L_{C_{8}, 1}, L_{E_{6}, 2}, L_{F_{4}, 3}$ and $L_{G_{2}, 4}$.

7) 10 simple modules. There are 9 affine VOAs whose numbers of inequivalent simple modules are $10 ; L_{A_{1}, 9}, L_{A_{2}, 3}, L_{A_{3}, 2}, L_{A_{9}, 1}, L_{B_{6}, 2}, L_{C_{2}, 3}, L_{C_{3}, 2}, L_{C_{9}, 1}$ and $L_{E_{8}, 4}$. 


\begin{tabular}{|c|c|c|c|c|c|c|c|}
\hline Information $\mathfrak{g}$ & $A_{1}$ & $A_{2}$ & $A_{5}$ & $C_{2}$ & $C_{5}$ & $E_{7}$ & $G_{2}$ \\
\hline Level & 5 & 2 & 1 & 2 & 1 & 2 & 3 \\
\hline Central charge & $15 / 7$ & $16 / 5$ & 5 & 4 & $55 / 7$ & $133 / 10$ & 6 \\
\hline $\operatorname{dim} \mathcal{X}_{\mathfrak{g}, k}$ & 6 & 4 & 4 & 6 & 6 & 6 & 6 \\
\hline Method & - & 0 & 0 & - & - & - & - \\
\hline NZWC & O & 0 & 0 & $x$ & 0 & O & $x$ \\
\hline
\end{tabular}

Table 5: 6 simple modules

\begin{tabular}{|c|c|c|c|c|}
\hline Information $\mathfrak{g}$ & $A_{1}$ & $A_{6}$ & $B_{3}$ & $C_{6}$ \\
\hline$\overline{\text { Level }}$ & $\overline{6} 6$ & $\overline{1}$ & $\overline{2}$ & $\bar{~} 1$ \\
\hline Central charge & $9 / 4$ & 6 & 6 & $39 / 4$ \\
\hline $\operatorname{dim} \mathcal{X}_{\mathfrak{g}, k}$ & 7 & 4 & 7 & 7 \\
\hline Method & - & 0 & - & - \\
\hline NZWC & O & 0 & $\times$ & 0 \\
\hline
\end{tabular}

Table 6: 7 simple modules

\begin{tabular}{|c|c|c|c|c|}
\hline Information $\mathfrak{g}$ & $A_{1}$ & $A_{7}$ & $B_{4}$ & $C_{7}$ \\
\hline 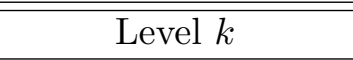 & $\overline{\overline{7}}$ & 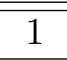 & 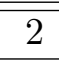 & $\overline{1} 1$ \\
\hline Central charge & $7 / 3$ & 7 & 8 & $35 / 3$ \\
\hline $\operatorname{dim} \mathcal{X}_{\mathfrak{g}, k}$ & 8 & 5 & 7 & 8 \\
\hline Method & - & 0 & $\diamond$ & - \\
\hline NZWC & O & $\mathrm{O}$ & $\mathrm{O}$ & O \\
\hline
\end{tabular}

Table 7: 8 simple modules

\begin{tabular}{|c|c|c|c|c|c|c|c|}
\hline Information $\mathfrak{g}$ & $A_{1}$ & $A_{8}$ & $B_{5}$ & $C_{8}$ & $E_{6}$ & $F_{4}$ & $G_{2}$ \\
\hline Level $k$ & 8 & $\overline{1}$ & 2 & 1 & 2 & 3 & 4 \\
\hline Central charge & $12 / 5$ & 8 & 10 & $68 / 5$ & $78 / 7$ & 13 & 7 \\
\hline $\operatorname{dim} \mathcal{X}_{\mathfrak{g}, k}$ & 9 & 5 & 9 & 9 & 6 & 9 & 9 \\
\hline Method & - & 0 & - & - & O & - & - \\
\hline NZWC & $\mathrm{O}$ & $\mathrm{O}$ & $x$ & O & O & $x$ & $x$ \\
\hline
\end{tabular}

Table 8: 9 simple modules 


\begin{tabular}{c|c|c|c|c|c|c|c|c|c}
\hline Information & $A_{1}$ & $A_{2}$ & $A_{3}$ & $A_{9}$ & $B_{6}$ & $C_{2}$ & $C_{3}$ & $C_{9}$ & $E_{8}$ \\
\hline \hline Level $k$ & 9 & 3 & 2 & 1 & 2 & 3 & 2 & 1 & 4 \\
\hline Central charge & $27 / 11$ & 4 & 5 & 9 & 12 & 5 & 7 & $171 / 11$ & $496 / 17$ \\
\hline $\operatorname{dim} \mathcal{X}_{\mathfrak{g}, k}$ & 10 & 6 & 7 & 6 & 10 & 10 & 10 & 10 & 10 \\
\hline Method & - & $\bigcirc$ & $\bigcirc$ & $\bigcirc$ & - & - & - & - & - \\
\hline NZWC & $\circ$ & $\times$ & $\times$ & $\bigcirc$ & $\times$ & $\times$ & $\times$ & $\bigcirc$ & $\times$ \\
\hline
\end{tabular}

Table 9: 10 simple modules

8) 11 simple modules. There are 5 affine VOAs whose numbers of inequivalent simple modules are $11 ; L_{A_{1}, 10}, L_{A_{10}, 1}, L_{B_{7}, 2}, L_{C_{10}, 1}$ and $L_{D_{4}, 2}$.

\begin{tabular}{|c|c|c|c|c|c|}
\hline Information $\mathfrak{g}$ & $A_{1}$ & $A_{10}$ & $B_{7}$ & $C_{10}$ & $D_{4}$ \\
\hline Level $k$ & 10 & 1 & 2 & 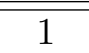 & 2 \\
\hline Central charge & $5 / 2$ & 10 & 14 & $35 / 2$ & 7 \\
\hline $\operatorname{dim} \mathcal{X}_{\mathfrak{g}, k}$ & 11 & 6 & 11 & 11 & 5 \\
\hline Method & - & O & - & - & $\mathrm{O}$ \\
\hline NZWC & O & O & $\times$ & O & O \\
\hline
\end{tabular}

Table 10: 11 simple modules

9) 12 simple modules. There are 7 affine VOAs whose numbers of inequivalent simple modules are $12 ; L_{A_{1}, 11}, L_{A_{11}, 1}, L_{B_{8}, 2}, L_{C_{11}, 1}, L_{D_{5}, 2}, L_{E_{7}, 3}$ and $L_{G_{2}, 5}$. The dimension of $\mathcal{X}_{B_{8}, 2}$ is determined in Proposition 18 in $\S 5$.

\begin{tabular}{c|c|c|c|c|c|c|c}
\hline Information & $A_{1}$ & $A_{11}$ & $B_{8}$ & $C_{11}$ & $D_{5}$ & $E_{7}$ & $G_{2}$ \\
\hline \hline Level $k$ & 11 & 1 & 2 & 1 & 2 & 3 & 5 \\
\hline Central charge & $33 / 13$ & 11 & 16 & $253 / 13$ & 9 & 19 & $70 / 9$ \\
\hline dim $\mathcal{X}_{\mathfrak{g}, k}$ & 12 & 7 & 12 & 12 & 9 & 12 & 12 \\
\hline Method & - & $\bigcirc$ & $\diamond$ & - & $\bigcirc$ & - & - \\
\hline NZWC & $\bigcirc$ & $\bigcirc$ & $\times$ & $\bigcirc$ & $\times$ & $\times$ & $\times$ \\
\hline
\end{tabular}

Table 11: 12 simple modules

10) 13 simple modules. There are 6 affine VOAs whose numbers of inequivalent simple modules are $13 ; L_{A_{1}, 12}, L_{A_{12}, 1}, L_{B_{3}, 3}, L_{B_{9}, 2}, L_{C_{12}, 1}$ and $L_{D_{6}, 2}$.

11) 14 simple modules. There are 5 affine VOAs whose numbers of inequivalent simple modules are $14 ; L_{A_{1}, 13}, L_{A_{13}, 1}, L_{B_{10}, 2}, L_{C_{13}, 1}$ and $L_{D_{7}, 2}$. 


\begin{tabular}{|c|c|c|c|c|c|c|}
\hline $\mathfrak{g}$ & $A_{1}$ & $A_{12}$ & $B_{3}$ & $B_{9}$ & $C_{12}$ & $D_{6}$ \\
\hline \hline Data & & & & & & \\
\hline Level $k$ & 12 & 1 & 3 & 2 & 1 & 2 \\
\hline $\operatorname{dim} \mathcal{X}_{\mathfrak{g}, k}$ & 13 & 7 & 13 & 13 & 13 & 10 \\
\hline Method & - & $\bigcirc$ & - & - & - & $\bigcirc$ \\
\hline NZWC & $\circ$ & $\bigcirc$ & $\times$ & $\times$ & $\bigcirc$ & $\times$ \\
\hline
\end{tabular}

Table 12: 13 simple modules

\begin{tabular}{|c|c|c|c|c|c|}
\hline Information $\mathfrak{g}$ & $A_{1}$ & $A_{13}$ & $B_{10}$ & $C_{13}$ & $D_{7}$ \\
\hline Level $k$ & $\overline{13}$ & $\overline{1}$ & $\overline{2}$ & $\overline{1}$ & 2 \\
\hline Central charge & $13 / 5$ & 13 & 20 & $117 / 5$ & 13 \\
\hline $\operatorname{dim} \mathcal{X}_{\mathfrak{g}, k}$ & 14 & 8 & 14 & 14 & 11 \\
\hline Method & - & O & - & - & 0 \\
\hline NZWC & O & O & $x$ & O & $\times$ \\
\hline
\end{tabular}

Table 13: 14 simple modules

12) 15 simple modules. There are 10 affine VOAs whose numbers of inequivalent simple modules are $15 ; L_{A_{1}, 14}, L_{A_{14}, 1}, L_{A_{2}, 4}, L_{A_{4}, 2}, L_{B_{11}, 2}, L_{C_{2}, 4}, L_{C_{14}, 1}, L_{C_{4}, 2}, L_{D_{8}, 2}$ and $L_{E_{8}, 5}$.

\begin{tabular}{c|c|c|c|c|c|c|c|c|c|c}
\hline Information & $A_{1}$ & $A_{14}$ & $A_{2}$ & $A_{4}$ & $B_{11}$ & $C_{2}$ & $C_{14}$ & $C_{4}$ & $D_{8}$ & $E_{8}$ \\
\hline \hline Level $k$ & 14 & 1 & 4 & 2 & 2 & 4 & 1 & 2 & 2 & 5 \\
\hline Central charge & $21 / 8$ & 14 & $32 / 7$ & $48 / 7$ & 22 & $40 / 7$ & $203 / 8$ & $72 / 7$ & 15 & $248 / 7$ \\
\hline $\operatorname{dim} \mathcal{X}_{\mathfrak{g}, k}$ & 15 & 8 & 9 & 9 & 15 & 15 & 15 & 15 & 12 & 15 \\
\hline Method & - & $\bigcirc$ & $\bigcirc$ & $\bigcirc$ & - & - & - & - & $\bigcirc$ & - \\
\hline NZWC & $\circ$ & $\circ$ & $\times$ & $\times$ & $\times$ & $\times$ & $\bigcirc$ & $\times$ & $\times$ & $\times$ \\
\hline
\end{tabular}

Table 14: 15 simple modules

13) 16 simple modules. There are 8 affine VOAs whose numbers of inequivalent simple modules are $16 ; L_{A_{1}, 15}, L_{A_{15}, 1}, L_{B_{12}, 2}, L_{B_{4}, 3}, L_{C_{15}, 1}, L_{D_{9}, 2}, L_{F_{4}, 4}$ and $L_{G_{2}, 6}$. The dimensions of $\mathcal{X}_{B_{12}, 2}$ and $\mathcal{X}_{B_{4}, 3}$ are determined in Propositions 19 and 17 in $\S 5$, respectively.

14) 17 simple modules. There are 5 affine VOAs whose numbers of inequivalent simple modules are $17 ; L_{A_{1}, 16}, L_{A_{16}, 1}, L_{B_{13}, 2}, L_{C_{16}, 1}$ and $L_{D_{10}, 2}$.

15) 18 simple modules. There are 5 affine VOAs whose numbers of inequivalent simple modules are $18 ; L_{A_{1}, 17}, L_{A_{17}, 1}, L_{B_{14}, 2}, L_{C_{17}, 1}$ and $L_{D_{11}, 2}$. 


\begin{tabular}{|c|c|c|c|c|c|c|c|c|}
\hline Information $\mathfrak{g}$ & $A_{1}$ & $A_{15}$ & $B_{12}$ & $B_{4}$ & $C_{15}$ & $D_{9}$ & $F_{4}$ & $G_{2}$ \\
\hline Level $k$ & 15 & $\overline{1} 1$ & 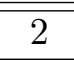 & 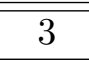 & $\overline{1} 1$ & 2 & 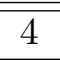 & 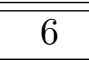 \\
\hline Central charge & $45 / 17$ & 15 & 24 & $54 / 5$ & $465 / 17$ & 17 & 16 & $42 / 5$ \\
\hline $\operatorname{dim} \mathcal{X}_{\mathfrak{g}, k}$ & 16 & 9 & 16 & 14 & 16 & 13 & 16 & 16 \\
\hline Method & - & O & $\diamond$ & $\diamond$ & - & $\mathrm{O}$ & - & - \\
\hline NZWC & O & 0 & $x$ & $x$ & O & $x$ & $x$ & $x$ \\
\hline
\end{tabular}

Table 15: 16 simple modules

\begin{tabular}{c|c|c|c|c|c}
\hline Information & $A_{1}$ & $A_{16}$ & $B_{13}$ & $C_{16}$ & $D_{10}$ \\
\hline \hline Level $k$ & 16 & 1 & 2 & 1 & 2 \\
\hline Central charge & $8 / 3$ & 16 & 26 & $88 / 3$ & 19 \\
\hline $\operatorname{dim} \mathcal{X}_{\mathfrak{g}, k}$ & 17 & 9 & 17 & 17 & 14 \\
\hline Method & - & $\bigcirc$ & - & - & $\bigcirc$ \\
\hline NZWC & $\bigcirc$ & $\bigcirc$ & $\times$ & $\bigcirc$ & $\times$ \\
\hline
\end{tabular}

Table 16: 17 simple modules

\begin{tabular}{c|c|c|c|c|c}
\hline Information & $A_{1}$ & $A_{17}$ & $B_{14}$ & $C_{17}$ & $D_{11}$ \\
\hline \hline Level $k$ & 17 & 1 & 2 & 1 & 2 \\
\hline Central charge & $51 / 19$ & 17 & 28 & $595 / 19$ & 21 \\
\hline dim $\mathcal{X}_{\mathfrak{g}, k}$ & 18 & 10 & 18 & 18 & 15 \\
\hline Method & - & $\bigcirc$ & - & - & $\bigcirc$ \\
\hline NZWC & $\circ$ & $\bigcirc$ & $\times$ & $\bigcirc$ & $\times$ \\
\hline
\end{tabular}

Table 17: 18 simple modules 
16) 19 simple modules. There are 6 affine VOAs whose numbers of inequivalent simple modules are $19 ; L_{A_{1}, 18}, L_{A_{18}, 1}, L_{B_{15}, 2}, L_{B_{5}, 3}, L_{C_{18}, 1}$ and $L_{D_{12}, 2}$. The dimension of $\mathcal{X}_{B_{5}, 3}$ is determined in Proposition 20 in $\S 5$. Non-zero Wronskian condition for $L_{B_{5}, 3}$ is not yet to be determined (see Remark in $\S 5.3$ ).

\begin{tabular}{|c|c|c|c|c|c|c|}
\hline Information $\mathfrak{g}$ & $A_{1}$ & $A_{18}$ & $B_{15}$ & $B_{5}$ & $C_{18}$ & $D_{12}$ \\
\hline Level $k$ & 18 & 1 & 2 & 3 & 1 & 2 \\
\hline Central charge & $27 / 10$ & 18 & 30 & $55 / 4$ & $333 / 10$ & 23 \\
\hline $\operatorname{dim} \mathcal{X}_{\mathfrak{g}, k}$ & 19 & 10 & 19 & 19 & 19 & 16 \\
\hline Method & - & O & - & $\diamond$ & - & - \\
\hline NZWC & O & 0 & $x$ & $?$ & 0 & $x$ \\
\hline
\end{tabular}

Table 18: 19 simple modules

17) 20 simple modules. There are 9 affine VOAs whose numbers of inequivalent simple modules are 20; $L_{A_{1}, 19}, L_{A_{19}, 1}, L_{A_{3}, 3}, L_{B_{16}, 2}, L_{C_{19}, 1}, L_{C_{3}, 2}, L_{D_{13}, 2}, L_{E_{6}, 3}$ and $L_{G_{2}, 7}$.

\begin{tabular}{c|c|c|c|c|c|c|c|c|c}
\hline \multirow{2}{*}{$A_{\text {Information }}$} & $A_{1}$ & \multirow{2}{*}{$A_{19}$} & $A_{3}$ & $B_{16}$ & $C_{19}$ & $C_{3}$ & $D_{13}$ & $E_{6}$ & $G_{2}$ \\
\hline \hline Level $k$ & 19 & 1 & 3 & 2 & 1 & 3 & 2 & 3 & 7 \\
\hline Central charge & $19 / 7$ & 19 & $45 / 7$ & 32 & $247 / 7$ & 9 & 25 & $78 / 5$ & $98 / 11$ \\
\hline $\operatorname{dim} \mathcal{X}_{\mathfrak{g}, k}$ & 20 & 11 & 13 & 20 & 20 & 20 & 17 & 12 & 20 \\
\hline Method & - & $\bigcirc$ & $\bigcirc$ & - & - & - & - & $\bigcirc$ & - \\
\hline NZWC & $\circ$ & $\circ$ & $\times$ & $\times$ & $\circ$ & $\times$ & $\times$ & $\times$ & $\times$ \\
\hline
\end{tabular}

Table 19: 20 simple modules

\section{The spaces of characters of some affine vertex operator algebras of type $B$}

In this section we determine the dimensions of spaces of characters of affine VOAs $L_{B_{\ell}, 2}(\ell=$ $4,8,12)$ and $L_{B_{\ell}, 3}(\ell=4,5)$. Since it seems that we cannot determine the dimensions of spaces of characters in this case by information of conformal weights and diagram automorphisms, we further use the techniques of $S$-matrix.

Let $P_{+}^{k}$ be a set of dominant integral weights of positive integral level $k$ of an affine VOA $L_{\mathfrak{g}, k}$. It is well-known $([9,8])$ that characters transform under $\tau \mapsto-1 / \tau$ as

$$
\operatorname{ch}_{\Lambda}(-1 / \tau)=\sum_{\Lambda^{\prime} \in P_{+}^{k}} S\left(\Lambda, \Lambda^{\prime}\right) \operatorname{ch}_{\Lambda^{\prime}}(\tau), \quad S\left(\Lambda, \Lambda^{\prime}\right) \in \mathbb{C} .
$$


Proposition 15 ([10, pp. 181][18, Proposition 4.19, pp. 236 (ii)]). Let $\Lambda, \Lambda^{\prime} \in P_{+}^{k}$ of $L_{B_{\ell}, k}$ and $\Lambda=\sum_{i=1}^{\ell} m_{i} \Lambda_{i}$ and $\Lambda^{\prime}=\sum_{i=1}^{\ell} n_{i} \Lambda_{i}$. Then

$$
S\left(\Lambda, \Lambda^{\prime}\right)=i^{\ell(\ell-1)} 2^{\ell-1}(k+2 \ell-1)^{-\ell / 2} \operatorname{det}\left(\sin \frac{2 \pi s_{i} t_{j}}{k+2 \ell-1}\right)_{1 \leq i, j \leq \ell},
$$

where

$$
s_{i}=\ell+\frac{1}{2}-i+\sum_{j=i}^{\ell-1} m_{j}+\frac{m_{\ell}}{2}, t_{i}=\ell+\frac{1}{2}-i+\sum_{j=i}^{\ell-1} n_{j}+\frac{n_{\ell}}{2}
$$

for $1 \leq i \leq \ell$. In particular, $S\left(\Lambda, \Lambda^{\prime}\right)$ for $L_{B_{\ell}, 2}$ is given in Table 20 , where we set $\lambda_{1}=\Lambda_{1}$,

\begin{tabular}{|c|c|c|c|c|c|}
\hline$\Lambda_{\Lambda} \Lambda^{\prime}$ & 0 & $2 \Lambda_{1}$ & $\Lambda_{\ell}$ & $\Lambda_{1}+\Lambda_{\ell}$ & $\lambda_{k}$ \\
\hline 0 & $\frac{1}{2 \sqrt{2 \ell+1}}$ & $\frac{1}{2 \sqrt{2 \ell+1}}$ & $\frac{1}{2}$ & $\frac{1}{2}$ & $\frac{1}{2 \ell+1}$ \\
\hline $2 \Lambda_{1}$ & $\frac{1}{2 \sqrt{2 \ell+1}}$ & $\frac{1}{2 \sqrt{2 \ell+1}}$ & $-\frac{1}{2}$ & $-\frac{1}{2}$ & $\frac{1}{2 \ell+1}$ \\
\hline$\Lambda_{\ell}$ & $\frac{1}{2}$ & $-\frac{1}{2}$ & $\frac{1}{2}$ & $-\frac{1}{2}$ & 0 \\
\hline$\Lambda_{1}+\Lambda_{\ell}$ & $\frac{1}{2}$ & $-\frac{1}{2}$ & $-\frac{1}{2}$ & $\frac{1}{2}$ & 0 \\
\hline$\lambda_{j}$ & $\frac{1}{\sqrt{2 \ell+1}}$ & $\frac{1}{\sqrt{2 \ell+1}}$ & 0 & 0 & $\frac{2}{\sqrt{2 \ell+1}} \cos \frac{2 \pi j k}{2 \ell+1}$ \\
\hline
\end{tabular}
$\lambda_{j}=\Lambda_{j}(2 \leq j \leq \ell-1), \lambda_{\ell}=2 \Lambda_{\ell}$.

Table 20: List of $S\left(\Lambda, \Lambda^{\prime}\right)$ for $L_{B_{\ell}, 2}$

\section{$5.1 \quad B_{4}$ of level 2 and 3}

We first determine the dimension of the space of characters of $L_{B_{4}, 2}$. The highest weights of simple $L_{B_{4}, 2}$-modules are given by $0, \Lambda_{1}, \Lambda_{2}, \Lambda_{3}, \Lambda_{4}, 2 \Lambda_{1}, 2 \Lambda_{4}, \Lambda_{1}+\Lambda_{4}$ whose conformal weights are respectively given by

$$
0, \frac{4}{9}, \frac{7}{9}, 1, \frac{1}{2}, 1, \frac{10}{9}, 1
$$

We prove a relation $\mathrm{ch}_{\Lambda_{3}}+\mathrm{ch}_{2 \Lambda_{1}}-\mathrm{ch}_{\Lambda_{1}+\Lambda_{4}}=0$. As being stated in Proposition 15 (also see [18, pp. 236 (ii)]) the $S$-transformations of characters $\operatorname{ch}_{\Lambda_{3}}, \operatorname{ch}_{\Lambda_{1}+\Lambda_{4}}, \operatorname{ch}_{2 \Lambda_{1}}$ are

$$
\begin{aligned}
& \operatorname{ch}_{\Lambda_{3}} \longrightarrow \frac{1}{3} \operatorname{ch}_{0}+\frac{1}{3} \operatorname{ch}_{2 \Lambda_{1}}-\frac{1}{3} \operatorname{ch}_{\Lambda_{1}}-\frac{1}{3} \operatorname{ch}_{\Lambda_{2}}+\frac{2}{3} \operatorname{ch}_{\Lambda_{3}}-\frac{1}{3} \operatorname{ch}_{2 \Lambda_{4}}, \\
& \operatorname{ch}_{\Lambda_{1}+\Lambda_{4}} \longrightarrow \frac{1}{2} \operatorname{ch}_{0}-\frac{1}{2} \operatorname{ch}_{2 \Lambda_{1}}-\frac{1}{2} \operatorname{ch}_{\Lambda_{4}}+\frac{1}{2} \operatorname{ch}_{\Lambda_{1}+\Lambda_{4}}, \\
& \operatorname{ch}_{2 \Lambda_{1}} \longrightarrow \frac{1}{6} \operatorname{ch}_{0}+\frac{1}{6} \operatorname{ch}_{2 \Lambda_{1}}-\frac{1}{2} \operatorname{ch}_{\Lambda_{4}}-\frac{1}{2} \operatorname{ch}_{\Lambda_{1}+\Lambda_{4}}+\frac{1}{3} \operatorname{ch}_{\Lambda_{1}}+\frac{1}{3} \operatorname{ch}_{\Lambda_{2}}+\frac{1}{3} \operatorname{ch}_{\Lambda_{3}}+\frac{1}{3} \operatorname{ch}_{2 \Lambda_{4}}
\end{aligned}
$$

It follows by (11)-(13) that $f(-1 / \tau)=f(\tau)$, where $f=\operatorname{ch}_{\Lambda_{3}}+\operatorname{ch}_{2 \Lambda_{1}}-\operatorname{ch}_{\Lambda_{1}+\Lambda_{4}}$. Since the central charge of $L_{B_{\ell}, 2}$ is 8 , the lowest power of $q$ in $f$ is not smaller than $2 / 3$. Therefore $f^{3}$ is a holomorphic cusp form of weight 0 , which shows $f=0$. 
Proposition 16. $\operatorname{dim} \mathcal{X}_{B_{4}, 2}=7$ and $\operatorname{ch}_{\Lambda_{3}}+\operatorname{ch}_{2 \Lambda_{1}}=\operatorname{ch}_{\Lambda_{1}+\Lambda_{4}}$. Moreover, $L_{B_{4}, 2}$ satisfies non-zero Wronskian condition.

Proof. The relation $\mathrm{ch}_{\Lambda_{3}}+\mathrm{ch}_{2 \Lambda_{1}}=\mathrm{ch}_{\Lambda_{1}+\Lambda_{4}}$ was already proved. By (10) and Lemma 4, it suffices to prove linear independence of $\operatorname{ch}_{\Lambda_{3}}$ and $\operatorname{ch}_{2 \Lambda_{1}}$. Suppose that $a \operatorname{ch}_{\Lambda_{3}}+b \mathrm{ch}_{2 \Lambda_{1}}=$ 0 . By Weyl's dimension formula, we have $84 a+44 b=0$. It follows from Proposition 15 that $S\left(\Lambda_{3}, \Lambda_{4}\right)=0$ and $S\left(2 \Lambda_{1}, \Lambda_{4}\right)=-1 / 2$. Since the conformal weight of $L_{B_{4}, 2}\left(\Lambda_{4}\right)$ is $1 / 2$ and $1 / 2+\mathbb{Z}_{>0}$ are not conformal weights, the coefficient of $q^{1 / 6}$ of the $S$-transformation of the linear relation yields $b / 2=0$. Therefore we have $a=b=0$.

We next show that $L_{B_{4}, 2}$ satisfies non-zero Wronskian condition. Since $\operatorname{ch}_{\Lambda_{3}}$ and $\operatorname{ch}_{2 \Lambda_{1}}$ have the same lowest powers, we have normalized forms of them as $q^{2 / 3}+\ldots$ and $q^{A-1 / 3}+\ldots$ with $A \in \mathbb{Z}_{>1}$. Then, by (10), Mason's inequality $42-12(3 / 2+A)=24-12 A \geq 0$ yields $A=$ 2 , which shows at the same time that $L_{B_{4}, 2}$ satisfies non-zero Wronskian condition.

The number of simple $L_{B_{4}, 3}$-modules is 16 . The conformal weights are $0,9 / 20,1$, $33 / 20,9 / 10,3 / 2,7 / 10,5 / 4,2 / 5,9 / 10,3 / 2,7 / 5,6 / 5,9 / 10,29 / 20,3 / 2$. The highest weights with conformal weight $9 / 10$ are $\Lambda_{3}, 2 \Lambda_{1}$ and $\Lambda_{1}+\Lambda_{4}$, and the highest weights with conformal weight $3 / 2$ are $3 \Lambda_{1}, \Lambda_{1}+2 \Lambda_{3}$ and $\Lambda_{3}+\Lambda_{4}$. Let $g=\operatorname{ch}_{\Lambda_{3}}+\operatorname{ch}_{2 \Lambda_{1}}-\operatorname{ch}_{\Lambda_{1}+\Lambda_{4}}$ and $h=\operatorname{ch}_{\Lambda_{3}+\Lambda_{4}}+\operatorname{ch}_{3 \Lambda_{1}}-\operatorname{ch}_{\Lambda_{1}+2 \Lambda_{4}}$, and we prove that $g=h=0$. By Proposition 15 it follows that

$$
g(-1 / \tau)=\sqrt{\frac{5-\sqrt{5}}{10}} g(\tau)+\sqrt{\frac{5+\sqrt{5}}{10}} h(\tau), \quad h(-1 / \tau)=\sqrt{\frac{5+\sqrt{5}}{10}} g(\tau)-\sqrt{\frac{5-\sqrt{5}}{10}} h(\tau) .
$$

Then ${ }^{t}(g, h)$ is a vector-valued modular form of weight 0 . Suppose that ${ }^{t}(g, h)$ is a non-zero vector-valued function. The lowest powers in $q$ of $g$ and $h$ are not smaller than 9/20 and $21 / 20$, respectively since the central charge of $L_{B_{4}, 3}$ is $54 / 5$. If both $g$ and $h$ are not zero, then $g$ and $h$ are linearly independent since their lowest powers of $q$ do not have an integral difference. We write the lowest powers of $q$ in them by $A \in 9 / 20+\mathbb{Z}_{\geq 0}$ and $B \in 21 / 20+\mathbb{Z}_{\geq 0}$, respectively. Then Mason's inequality is equivalent to $2-12(9 / 20+21 / 20+A+B)=$ $-4(4+3 A+3 B) \geq 0$, which is a contradiction. If $h=0$, then $g \neq 0$ and the lowest power in $q$ of $g$ is positive, which contradicts to Mason's inequality. Therefore, we have $g=h=0$, that is, $\operatorname{ch}_{\Lambda_{3}}+\operatorname{ch}_{2 \Lambda_{1}}=\operatorname{ch}_{\Lambda_{1}+\Lambda_{4}}$ and $\operatorname{ch}_{\Lambda_{3}+\Lambda_{4}}+\operatorname{ch}_{3 \Lambda_{1}}=\operatorname{ch}_{\Lambda_{1}+2 \Lambda_{4}}$.

Proposition 17. $\operatorname{dim} \mathcal{X}_{B_{4}, 3}=14$ and $\operatorname{ch}_{\Lambda_{3}}+\operatorname{ch}_{2 \Lambda_{1}}=\operatorname{ch}_{\Lambda_{1}+\Lambda_{4}}, \operatorname{ch}_{\Lambda_{3}+\Lambda_{4}}+\operatorname{ch}_{3 \Lambda_{1}}=\operatorname{ch}_{\Lambda_{1}+2 \Lambda_{4}}$. Non-zero Wronskian condition does not hold.

Proof. We prove that both sets $\left\{\operatorname{ch}_{\Lambda_{3}}, \operatorname{ch}_{2 \Lambda_{1}}\right\}$ and $\left\{\operatorname{ch}_{\Lambda_{3}+\Lambda_{4}}, \operatorname{ch}_{3 \Lambda_{1}}\right\}$ are linearly independent. Suppose that $a \operatorname{ch}_{\Lambda_{3}}+b \operatorname{ch}_{2 \Lambda_{1}}=0$ with some $a, b \in \mathbb{C}$. By Weyl's dimension formula, the coefficient of $q^{9 / 20}$ is $84 a+44 b=0$. Then by Proposition 15 , we have $S\left(\Lambda_{3}, \Lambda_{4}\right)=1 / 2 \sqrt{5}$ and $S\left(2 \Lambda_{1}, \Lambda_{4}\right)=-1 / 2 \sqrt{5}$. Since the conformal weight of $L_{B_{4}, 3}\left(\Lambda_{4}\right)$ is $9 / 20$ and any number in the set $9 / 20+\mathbb{Z}_{>0}$ is not a conformal weight, the coefficient of $q^{0}(=1)$ of the $S$-transformation of the relation $a \operatorname{ch}_{\Lambda_{3}}+b \mathrm{ch}_{2 \Lambda_{1}}=0$ is $a / 2 \sqrt{5}-b / 2 \sqrt{5}=0$. Thus we have $a=b=0$. Also suppose that $c \operatorname{ch}_{\Lambda_{3}+\Lambda_{4}}+d \operatorname{ch}_{3 \Lambda_{1}}=0$ with $c, d \in \mathbb{C}$. Then the same discussions given above show $c=d=0$.

The normalized forms of $\operatorname{ch}_{\Lambda_{3}}$ and $\operatorname{ch}_{2 \Lambda_{1}}$ are $q^{9 / 20}+\cdots$ and $q^{9 / 20+A}+\cdots$ with $A-9 / 20 \in$ $\mathbb{Z}_{>0}$, and ones of $\operatorname{ch}_{\Lambda_{3}+\Lambda_{4}}$ and $\operatorname{ch}_{3 \Lambda_{1}}$ are $q^{21 / 20}+\cdots$ and $q^{B+21 / 20}+\cdots$ with $B-21 / 20 \in \mathbb{Z}_{>0}$. 
Then by Mason's inequality, we have $-4(3 A+3 B-26) \geq 0$. Setting $A=(9+20 \mathrm{~m}) / 20$ and $B=(21+20 n) / 20$ for positive integers $m$ and $n$, we have $3 m+3 n \leq 43 / 2$. Therefore, non-zero Wronskian condition does not hold, i.e. there are no $m$ and $n$ such that $3(m+n)=$ $43 / 2$.

\section{$5.2 \quad B_{8}$ and $B_{12}$ of level 2}

The number of inequivalent simple $L_{B_{8}, 2}$-modules is 12 , the central charge of $L_{B_{8}, 2}$ is 16 and the conformal weights of simple $L_{B_{8}, 2}$-modules are

$$
0,1, \frac{36}{17}, \frac{35}{17}, \frac{33}{17}, \frac{30}{17}, \frac{26}{17}, \frac{21}{17}, \frac{15}{17}, \frac{8}{17}, \frac{3}{2}, 1,
$$

where the highest weights of simple modules with conformal weight 1 are $\Lambda_{8}$ and $2 \Lambda_{1}$.

Proposition 18. $\operatorname{dim} \mathcal{X}_{B_{8}, 2}=12$ and $L_{B_{8}, 2}$ does not satisfy non-zero Wronskian condition.

Proof. By Lemma 4 and (14), it suffices to prove $\mathrm{ch}_{\Lambda_{8}}$ and $\mathrm{ch}_{2 \Lambda_{1}}$ are linearly independent. Suppose that $a \operatorname{ch}_{\Lambda_{8}}+b \mathrm{ch}_{2 \Lambda_{1}}=0$. By Weyl's dimension formula, the coefficient of $q^{1 / 3}$ of the linear relation is $256 a+152 b=0$ and it follows from Proposition 15 , we have $S\left(\Lambda_{8}, 0\right)=$ $1 / 2 \sqrt{17}$ and $S\left(2 \Lambda_{1}, 0\right)=1 / 2$. Applying $S$-transformation and taking the coefficient of $q^{-2 / 3}$ of the linear relation we have $a / 2 \sqrt{17}+b / 2=0$, and hence $a=b=0$.

The normalization of $\operatorname{ch}_{\Lambda_{8}}$ and $\operatorname{ch}_{2 \Lambda_{1}}$ are $q^{1 / 3}+\cdots$ and $q^{A-2 / 3}+\cdots$ with $A \in \mathbb{Z}_{>0}$. Then Mason's inequality implies $54-12 A \geq 0$, which shows non-zero Wronskian condition failed.

We next determine $\operatorname{dim} \mathcal{X}_{B_{12}, 2}$. The number of simple $L_{B_{12}, 2}$-modules is 16 and the central charge of $L_{B_{12}, 2}$ is 24 . The conformal weights of simple $L_{B_{12}, 2}$-modules are $0,12 / 25$, $23 / 25,1,33 / 25,3 / 2,42 / 25,2,2,57 / 25,63 / 25,68 / 25,72 / 25,3,77 / 25,78 / 25$ where the highest weights of simple modules with conformal weight 2 are $\Lambda_{5}$ and $\Lambda_{1}+\Lambda_{12}$.

Proposition 19. $\operatorname{dim} \mathcal{X}_{B_{12}, 2}=16$ and $L_{B_{12}, 2}$ does not satisfy non-zero Wronskian condition.

Proof. It is enough to prove that $\operatorname{ch}_{\Lambda_{5}}$ and $\operatorname{ch}_{\Lambda_{1}+\Lambda_{12}}$ are linearly independent. Suppose that $a \operatorname{ch}_{\Lambda_{5}}+b \operatorname{ch}_{\Lambda_{1}+\Lambda_{12}}=0$ with some $a, b \in \mathbb{C}$. By Weyl's dimension formula, we have $53130 a+98304 b=0$. It follows from Proposition 15 that $S\left(\Lambda_{5}, \Lambda_{12}\right)=0$ and $S\left(\Lambda_{1}+\right.$ $\left.\Lambda_{12}, \Lambda_{12}\right)=-1 / 2$. Since the conformal weight of $L_{B_{12}, 2}\left(\Lambda_{12}\right)$ is $3 / 2$, and any number in the set $3 / 2+\mathbb{Z}_{>0}$ is not a conformal weight, taking the coefficient of $q^{1 / 2}$ of the $S$-transformation of the linear relation gives $-b / 2=0$, which yields $a=b=0$. The normalization of $\operatorname{ch}_{\Lambda_{5}}$ and $\operatorname{ch}_{\Lambda_{1}+\Lambda_{12}}$ are $1+\cdots$ and $q^{A-1}+\ldots$ with $A \in \mathbb{Z}_{>3}$ since there is a simple module $L_{B_{12}, 2}\left(\Lambda_{10}\right)$ with conformal weight 3 . Thus Mason's inequality yields $90-12 A \geq 0$, which shows non-zero Wronskian condition failed.

\section{$5.3 \quad B_{5}$ of level 3}

The number of simple $L_{B_{5}, 3}$-modules is 19 and its central charge is $55 / 4$. The conformal weights of simple modules are $0,5 / 12,55 / 96,3 / 4,11 / 12,1,33 / 32,7 / 6,5 / 4,5 / 4,45 / 32,3 / 2$, $3 / 2,151 / 96,5 / 3,163 / 96,7 / 4,61 / 32,65 / 32$. The highest weights of simple modules with conformal weights $5 / 4$ and $3 / 2$ are $2 \Lambda_{5}, \Lambda_{1}+\Lambda_{2}$, and $3 \Lambda_{1}, \Lambda_{1}+\Lambda_{3}$, respectively. 
Proposition 20. $\operatorname{dim} \mathcal{X}_{B_{5}, 3}=19$.

Proof. It is enough to prove that $\left\{\operatorname{ch}_{2 \Lambda_{5}}, \operatorname{ch}_{\Lambda_{1}+\Lambda_{2}}\right\}$ and $\left\{\operatorname{ch}_{3 \Lambda_{1}}, \operatorname{ch}_{\Lambda_{1}+\Lambda_{3}}\right\}$ are linearly independent. Suppose that $a \mathrm{ch}_{2 \Lambda_{5}}+b \mathrm{ch}_{\Lambda_{1}+\Lambda_{2}}=0$. By Weyl's dimension formula, the coefficient of $q^{5 / 4-55 / 96}\left(=q^{65 / 96}\right)$ of both sides gives $462 a+429 b=0$. By Proposition 15, we have $S\left(2 \Lambda_{5}, \Lambda_{5}\right)=1 / 2 \sqrt{6}$ and $S\left(\Lambda_{1}+\Lambda_{2}, \Lambda_{5}\right)=-1 / 2 \sqrt{2}$. Since the conformal weight of $L_{B_{5}, 3}\left(\Lambda_{5}\right)$ is $55 / 96$ and any number in the set $55 / 96+\mathbb{Z}_{<0}$ is not a conformal weight, the coefficient of $q^{0}(=1)$ of the $S$-transformation of the relation is $a / 2 \sqrt{6}-b / 2 \sqrt{2}=0$. Therefore we have $a=b=0$. Suppose that $c \operatorname{ch}_{3 \Lambda_{1}}+d \operatorname{ch}_{\Lambda_{1}+\Lambda_{3}}=0$ with $c, d \in \mathbb{C}$. The same discussion above shows that $c=d=0$.

Remark. Non-zero Wronskian condition for $L_{B_{5}, 3}$ does not follow directly. The normalization of the characters $\operatorname{ch}_{2 \Lambda_{5}}$ and $\operatorname{ch}_{\Lambda_{1}+\Lambda_{2}}$ are $q^{5 / 4-55 / 96}+\cdots$ and $q^{5 / 4+A-55 / 96}+\cdots$ with $A \in$ $\mathbb{Z}_{>0}$, and ones of $\operatorname{ch}_{3 \Lambda_{1}}$ and $\operatorname{ch}_{\Lambda_{1}+\Lambda_{3}}$ are $q^{3 / 2-55 / 96}+\cdots$ and $q^{3 / 2+B-55 / 96}$ with $B \in \mathbb{Z}_{>0}$. Then Mason's inequality implies $-12(-16+A+B) \geq 0$. It seems to be hard to determine $A$ and $B$ without knowing coefficients of higher power of $q$.

\section{Acknowledgment}

The authors wish to express their thanks to G. Mason for several helpful comments concerning to vector-valued modular forms and modular linear differential equations. Y. A. was supported by Japan Society for Promotion of Science, Grant-in-Aid for Young Scientists (B) 25800003. M. K. was supported by Japan Society for the Promotion of Science, Grant-inAid for Scientific Research (B) 23340010. K. N. was supported by Japan Society for the Promotion of Science, Grant-in-Aid for Challenging Exploratory Research 26610007. The second author was also partially supported by Max-Planck Institute for Mathematics and International Center of Theoretical Physics.

\section{References}

1. Deligne, P.: La série exceptionnalle de groupes de Lie. C. R. Acad. Sci. Paris Sr. I Math. 322321326 (1996).

2. Dong, C., Li, H., and Mason, G.: Modular-invariance of trace functions in orbifold theory and generalized Moonshine, Commun. Math. Phys. 214 1-56 (2000)

3. Eguchi, T. and Ooguri, H.: Differential equations for conformal characters in moduli space, Physics Letters B 203, 44-46 (1988)

4. Eguchi, T. and Ooguri, H.: Differential equations for characters of Virasoro and affine Lie algebras, Nuclear Phys. B 313, 492-508 (1989)

5. Frenkel, I. and Zhu, Y.: Vertex operator algebras associated to representations of affine and Virasoro algebras, Duke Math. J. 66 No. 1 123-168 (1992)

6. Fuchs, J., Schellekens, B. and Schweigert, C.: From Dynkin diagram symmetries to fixed point structures, Commun. Math. Phys. 180 39-97 (1996) 
7. Humphreys, J. E.: Introduction to Lie algebras and representation theory, Graduate Texts in Mathematics, Vol. 9, Springer-Verlag, New York-Berlin (1978)

8. Kac, V. G.: Infinite dimensional Lie algebras, 3rd edition, Cambridge University Press, Cambridge (1990)

9. Kac, V. G. and Peterson, D. H.: Infinite-dimensional Lie algebras, theta functions and modular forms, Adv. Math. 53 125-264 (1984)

10. Kac, V. G. and Wakimoto, M.: Modular and conformal invariance constraints in representation theory of affine algebras, Adv. Math. 70 156-236 (1988)

11. Kaneko, M., Nagatomo, K. and Sakai, Y.: Modular forms and second order ordinary differential equations: applications to vertex operator algebras. Lett. Math. Phys. 103 no. 4 439-453 (2013)

12. Kaneko, M. and Zagier, D.: Supersingular $j$-invariants, hypergeometric series, and Atkin's orthogonal polynomials, AMS/IP Studies in Advanced Mathematics, 7 97-126 (1998)

13. Marks, C.: Irreducible vector-valued modular forms of dimension less than six, Illinois Journal of Mathematics 55 No. 4 1267-1297 (2011)

14. Mason, G.: Vector-valued modular forms and linear differential operators, Int. J. Number Theory, 3 no. 3 377-390 (2007)

15. Mathur, S. D., Mukhi, S. and Sen A.: On the classification of rational conformal field theories, Phys. Letter B 213 No. 3 303-308 (1988)

16. Milas, A.: Ramanujan's "Lost Notebook" and the Virasoro algebra, Commun. Math. Phys. 251 567-588 (2004)

17. Milas, A.: Virasoro algebra, Dedekind $\eta$-functions and specialized Macdonald identities, Transformation Groups 9, No. 3. 273-288 (2004)

18. Wakimoto, M.: Infinite-dimensional Lie algebras, Translated from the 1999 japanese original by Kenji Iohara. Translations of Mathematical monographs 195 Iwanami Series in Modern Mathematics. American Mathematical Society, Providence (2001)

19. Zhu, Y.: Modular invariance of characters of vertex operator algebras, Journal of the American Mathematical Society 9 No. 1 237-302 (1996) 